\title{
A Fully Coupled Turbulent Low-Speed Preconditioner for Harmonic Balance Applications ${ }^{\text {th }}$
}

\author{
Reza Djeddi ${ }^{\mathrm{a}, *}$, Jason Howison $^{\mathrm{b}}$, Kivanc Ekici $^{\mathrm{c}}$ \\ ${ }^{a}$ Graduate Student, Department of Mechanical, Aerospace and Biomedical Engineering. \\ University of Tennessee, Knoxville \\ ${ }^{b}$ Assistant Professor, The Citadel School of Engineering \\ ${ }^{c}$ Associate Professor, Department of Mechanical, Aerospace and Biomedical Engineering. \\ University of Tennessee, Knoxville
}

\begin{abstract}
Aeromechanical design of wind turbines requires a high-fidelity analysis toolbox that can cover a wide spectrum of flow speeds from incompressible to compressible flow regimes. As it is known, the convergence of the compressible densitybased flow solvers is deteriorated when the flow lies in an essentially incompressible regime due to the disparity between the eigenvalues of the flux Jacobian matrices. This problem is usually resolved using a low-speed preconditioner that can balance the eigenvalues, thus alleviating the convergence issues in the densitybased flow solver. In this paper, a low-speed preconditioner that fully couples the Reynolds-averaged Navier-Stokes equations with the one equation SpalartAllmaras turbulence model is developed for steady as well as unsteady flows. The preconditioner incorporates the working variable of the turbulence model into the formulation, thus resulting in balanced artificial dissipation terms and modified local time-steps to ensure convergence acceleration for the compressible flow solver. The preconditioner is first applied to a set of two-dimensional steady flow cases to assess the convergence acceleration. This new preconditioner is further enhanced with an unsteady limiter that improves the convergence of the harmonic balance solver used for the simulation of unsteady periodic flows such as those that arise in wind turbine applications. Although the CPU time per iteration is increased by about $9 \%-11 \%$, the present preconditioner is capable of reducing the number of required iterations by about $50 \%-60 \%$ in order to achieve the same level of accuracy as with the non-preconditioned solver.
\end{abstract}

Keywords: Low-Speed Preconditioning, Spalart-Allmaras Turbulence Model, Harmonic Balance, Unsteady Flows, Wind Turbine

\footnotetext{
औ An earlier version of this paper was presented as Paper No. 3202 at the 22nd AIAA Computational Fluid Dynamics Conference (Dallas, TX, 22-26 June 2015).

* Corresponding Author

Email addresses: djeddi@utk.edu (Reza Djeddi), jhowison@citadel.edu (Jason

Howison), ekici@utk.edu (Kivanc Ekici)
}

Preprint submitted to Aerospace Science and Technology

February 16, 2016

(C) 2016. This manuscript version is made available under the Elsevier user license http://www.elsevier.com/open-access/userlicense/1.0/ 


\section{Nomenclature}

$\begin{array}{ll}b & \text { half-chord length of the airfoil } \\ c & \text { speed of sound } \\ E & \text { total energy } \\ \mathbf{F}, \mathbf{G} & \text { flux vectors } \\ H & \text { total enthalpy } \\ k & \text { reduced frequency, } k=b \omega / U_{\infty} \\ p & \text { pressure } \\ P r_{l} & \text { laminar Prandtl number } \\ P r_{t} & \text { turbulent Prandtl number } \\ S_{t} & \text { source term for the turbulence model } \\ t & \text { time } \\ \mathbf{U} & \text { vector of conservative variables } \\ \mathbf{U}_{0} & \text { vector of primitive variables } \\ u, v & \text { Cartesian velocities } \\ U_{\infty} & \text { free stream velocity } \\ \mathcal{V} & \text { control volume } \\ x, y & \text { Cartesian coordinates } \\ \xi, \eta & \text { cross-flow coordinates } \\ \gamma & \text { specific heat ratio } \\ \mu_{l}, \mu_{t} & \text { laminar and turbulent dynamic viscosity } \\ \nu & \text { kinematic viscosity } \\ \tilde{\nu} & \text { working variable for Spalart-Allmaras turbulence model } \\ \rho & \text { density } \\ \tau & \text { pseudo-time } \\ & \end{array}$

\section{Introduction}

The aeromechanical design of wind turbines is a complex and multidisciplinary task. This process requires not only the consideration of a very large number of operating regimes of very low to moderate Mach number flows with low and high turbulence intensities, but also the applicability to conventional and prospective unconventional new designs subject to challenging off-design conditions. This necessitates a high-fidelity, robust flow solver that can cover a wide spectrum of flow speeds and parameter space. In incompressible regimes, such as those seen in wind turbine applications where the density variations become small as the Mach number approaches zero, the flow field can be computed using a compressible solver with the aid of a "low-speed preconditioner." Various efforts have been made which are presented in the literature by Turkel $[1,2,3]$, Choi and Merkle [4], Weiss and Smith [5], and Van Leer [6] with varying degrees of success and a multitude of simplifications and limitations that are imposed along the way, especially in viscous cases.

Traditionally, the Navier-Stokes equations and the turbulence model equation(s) are treated in a segregated fashion, i.e. the turbulence model is not fully 
coupled to the governing equations and its solution is lagged. This approach allows one to discretize the turbulence equations using a lower-order upwind scheme thus eliminating the need for an artificial dissipation while providing extra stability [7]. Another advantage of this approach is that the turbulent variable(s) is/are not required to be included in the low-speed preconditioner formulations. On the other hand, solving the Navier-Stokes and turbulence model equations in a fully coupled fashion has the advantages of providing higher accuracy while being easier to implement into an existing solver. However, this approach can also hinder the convergence rate when dealing with low-speed regimes of flow.

The direct treatment of the turbulence model in low-speed preconditioning formulations were mostly ignored in previous works reported in literature. As an exception to this, Venkateswaran and Merkle [8] incorporated the $k-\epsilon$ turbulence model into their low-speed preconditioner for the analysis of reacting turbulent flows and non-reacting flows in a mixing layer. However, due to the presence of the turbulent kinetic energy, $k$, in the pressure gradient term and the inter-relation of the $k$ and $\epsilon$ equations, the eigenvalues of the coupled preconditioning system turn out to be complex-valued. This will be undesirable since the system would no longer be strictly hyperbolic, making it ill-posed for explicit time marching schemes. As shown in [8], the effective speed of sound can be introduced where a "turbulent" pressure is defined as a function of the original pressure, the turbulent kinetic energy and density. Recently, a similar approach has been used by Campobasso et al. [9] for time-accurate solution of horizontal-axis wind turbine (HAWT) turbulent flows using a $k-\omega$ shear-stress transport (SST) turbulence model. However, the application of the preconditioning to the steady and unsteady RANS equations that are fully coupled to the Spalart-Allmaras turbulence model, on the other hand, has not been investigated. Note that, when fully coupled to the RANS equations, the one equation Spalart-Allmaras turbulence model does not produce complex eigenvalues for the flux Jacobians thus eliminating the need to define a "turbulent" pressure term.

In the literature, almost all low-speed preconditioning tools are designed to pre-multiply the time derivatives by a preconditioning matrix to overcome the stiffness problem of the system of equations. Also, more often, a set of primitive variables is used to construct this matrix since it has been proven that it can ease the derivation process. The differences are then in terms of the preconditioning matrix and the set of primitive variables used. In a recent effort, Colin et al. [10] have studied a robust low-speed preconditioning tool for viscous/turbulent flows based on the symmetric preconditioner of Weiss and Smith [5] and the viscous preconditioner of Choi and Merkle [4] in a combined form of the so-called WSCM preconditioner. Also, Campobasso and Baba-Ahmadi [11] have used a slightly different viscous preconditioner that originated from the work of Venkateswaran and Merkle [8] and applied it to unsteady flow past horizontal axis wind turbine blades. More recently, Howison and Ekici [12] used an inviscid preconditioner based on Turkel's method [3] for unsteady harmonic balance analysis of wind turbine flows. 
Due to the fact that most of the fundamental wind turbine unsteady problems are periodic in time, frequency-domain techniques can be utilized to avoid prohibitively costly computational effort of time-domain RANS solvers. The application of a harmonic balance (HB) method $[13,14]$ to Navier-Stokes (NS) solvers has proven to be a promising tool for turbomachinery and wind turbine flow problems $[15,11,12,16,17]$. It is worth mentioning that higher harmonics can be introduced in the flow field due to the wind turbine tower shadow which necessitates the use of large number of sub-time levels in the harmonic balance solver. The aforementioned HB-NS solver can be enhanced with the one-equation Spalart-Allmaras [18] turbulence model that incorporates a rotation correction reducing the eddy viscosity in the vicinity of regions where the vorticity surpasses the strain rate $[19,20]$.

The spatial discretization of the convective and diffusive terms includes the central difference formula that can be stabilized and further enhanced with an artificial or numerical dissipation that uses a blend of second and fourth differences $[21,22,23]$. One main difference between wind turbine flows and other aerodynamic problems for which compressible harmonic balance RANS solvers have been used is that the flow-field for wind turbines falls in the incompressible regime. This entails the use of a robust low-speed preconditioning to ensure the accuracy of the solutions in a wide range of flow speeds while guaranteeing a rapid convergence at each sub-time level of the harmonic balance RANS solver.

In this paper, we develop a fully coupled viscous low-speed preconditioning that incorporates the Spalart-Allmaras turbulence model. This approach appears to alleviate the convergence problems that are inherited from applying a compressible solver to an essentially incompressible flow. The preconditioner couples the turbulence model to the rest of the governing equations to ensure the correct scaling of the turbulence equation and its respective artificial dissipation term that improves the convergence rate and also the accuracy of the compressible solver in low-subsonic flow regimes. The preconditioning is further enhanced with a mixed-mechanism that deals with the unsteadiness of the fluid flow through the correct modification of the preconditioning matrix for the harmonic balance implementation.

As mentioned earlier, although the $k-\epsilon$ and $k-\omega$ SST models have been used in conjunction with the low-speed preconditioner in a fully coupled fashion $[8,9]$, there has been no application of the idea to one-equation turbulence models. Therefore, to the best of our knowledge, this is the first work reported in the literature that presents a viscous low-speed preconditioner that incorporates Spalart-Allmaras turbulence model into its formulation. It is also the first time this technique is used in conjunction with the harmonic balance technique for the simulation of unsteady periodic flows using the one-equation Spalart-Allmaras turbulence model. The fully coupled viscous low-speed preconditioner is first applied to a set of steady and fully turbulent flow cases including the turbulent flow over a flat plate with zero pressure gradient and the low-speed flow over the S809 airfoil. Next, the method is applied to unsteady pitching airfoil cases to assess the effectiveness of the preconditioner with unsteady limiters (mixed-mechanism) in dealing with unsteady low-speed flow regimes. 


\section{Governing Equations and Mathematical Formulation}

Unsteady flow past the wind turbine airfoils is governed by the Reynoldsaveraged Navier-Stokes equations which include the modified Spalart-Allmaras turbulence model $[19,20]$. Note that this version of the Navier-Stokes equations assumes Newtonian fluid where the viscous stresses are linearly proportional to the strain rate. The two-dimensional conservation form of the governing equations for a moving grid becomes:

$$
\frac{\partial \mathbf{U}}{\partial t}+\frac{\partial \mathbf{F}}{\partial x}+\frac{\partial \mathbf{G}}{\partial y}=\mathbf{S}
$$

where the vector of conservative variables is $\mathbf{U}=[\rho, \rho u, \rho v, \rho E, \rho \tilde{\nu}]^{T}$ and the vectors of inviscid and viscous fluxes in $x$ - and $y$-directions, $\mathbf{F}$ and $\mathbf{G}$, and the vector of source terms, $\mathbf{S}$, are defined as:

$\mathbf{F}=\left[\begin{array}{c}\rho u-\rho u_{g} \\ \rho u^{2}+p-\tau_{x x}-\rho u u_{g} \\ \rho u v-\tau_{x y}-\rho v u_{g} \\ \rho u h-\tau_{x h}-\rho E u_{g} \\ \rho u \tilde{\nu}-\tau_{x \nu}-\rho \tilde{\nu} u_{g}\end{array}\right], \mathbf{G}=\left[\begin{array}{c}\rho v-\rho v_{g} \\ \rho u v-\tau_{y x}-\rho u v_{g} \\ \rho v^{2}+p-\tau_{y y}-\rho v v_{g} \\ \rho v h-\tau_{y h}-\rho E v_{g} \\ \rho v \tilde{\nu}-\tau_{y \nu}-\rho \tilde{\nu} v_{g}\end{array}\right], \mathbf{S}=\left[\begin{array}{c}0 \\ 0 \\ 0 \\ 0 \\ S_{t}\end{array}\right]$

Also, $\tilde{\nu}$ is the viscosity-like working variable of the Spalart-Allmaras turbulence model [18]. In general, the grid motion velocity is incorporated into the governing equations by $u_{g}$ and $v_{g}$ in $x$ and $y$ directions, respectively. The pressure $p$ and specific enthalpy $h$ are defined in terms of the conservative variables based on the assumption of an ideal gas with a constant specific heat ratio as:

$$
\begin{gathered}
p=(\gamma-1) \rho\left[E-\frac{1}{2}\left(u^{2}+v^{2}\right)\right] \\
h=\frac{\rho E+p}{\rho}=\frac{\gamma}{\gamma-1} \frac{p}{\rho}+\frac{1}{2}\left(u^{2}+v^{2}\right)
\end{gathered}
$$

where the flow is adiabatic and the inviscid fluxes depend on the conservative variables $\mathbf{U}$, viscous fluxes depend on the gradients of the flow velocities, temperature and the working variable of the turbulence model as defined below for the shear stress terms of the Navier-Stokes equations: 


$$
\begin{gathered}
\tau_{x x}=\left(\mu_{l}+\mu_{t}\right)\left(\frac{4}{3} \frac{\partial u}{\partial x}-\frac{2}{3} \frac{\partial v}{\partial y}\right) \\
\tau_{y y}=\left(\mu_{l}+\mu_{t}\right)\left(\frac{4}{3} \frac{\partial v}{\partial y}-\frac{2}{3} \frac{\partial u}{\partial x}\right) \\
\tau_{x y}=\tau_{y x}=\left(\mu_{l}+\mu_{t}\right)\left(\frac{\partial u}{\partial y}-\frac{\partial v}{\partial x}\right) \\
\tau_{x H}=u \tau_{x x}+v \tau_{x y}+\left(\frac{\mu_{l}}{P r_{l}}+\frac{\mu_{t}}{P r_{t}}\right) \frac{\partial H}{\partial x} \\
\tau_{y H}=u \tau_{x y}+v \tau_{y y}+\left(\frac{\mu_{l}}{P r_{l}}+\frac{\mu_{t}}{P r_{t}}\right) \frac{\partial H}{\partial y}
\end{gathered}
$$

In the above equations, the $\frac{2}{3}$ and $\frac{4}{3}$ terms come from the Stokes' hypothesis where the second coefficient of viscosity (dilational), $\lambda$, is proportional to the dynamic coefficient of viscosity (shear), $\mu$, such that $\lambda+\frac{2}{3} \mu=0$. Also, $\mu_{l}$ and $\mu_{t}$ are the laminar and eddy viscosities, respectively, where the former is defined based on the Sutherland's law and the latter is determined using the Spalart-Allmaras turbulence model $[18,19,20]$. The turbulent viscosity source term, $S_{t}$, in Equation (1) is given by

$$
S_{t}=\mathcal{P}-\mathcal{D}+c_{b 2}(\nabla \tilde{\nu})^{2}
$$

where the production, $\mathcal{P}$, and wall destruction, $\mathcal{D}$, terms are defined as

$$
\mathcal{P}=c_{b 1} \tilde{S} \tilde{\nu}, \quad \mathcal{D}=\left(c_{w 1} f_{w}\right)\left[\frac{\tilde{\nu}}{d}\right]^{2}
$$

with $\tilde{S}$ denoting the modified vorticity, i.e.,

$$
\tilde{S}=S+\frac{\tilde{\nu}}{\kappa^{2} d^{2}} f_{v 2}, \quad f_{v 2}=1-\frac{\chi}{1+\chi f_{v 1}}
$$

where $S$ is the magnitude of the vorticity, and $d$ is the distance to the closest wall. Also, the turbulent viscosity $\mu_{t}$ is defined by the standard Spalart-Allmaras turbulence model [18] as

$$
\mu_{t}=\rho \tilde{\nu} f_{v 1}(\chi)
$$

where

$$
f_{v 1}(\chi)=\frac{\chi^{3}}{\chi^{3}+c_{v 1}^{3}}, \quad \chi=\frac{\mu_{t}}{\mu_{l}}
$$

The function $f_{w}$ is:

$$
f_{w}=g\left[\frac{1+c_{w 3}^{6}}{g^{6}+c_{w 3}^{6}}\right]^{1 / 6}, \quad g=r+c_{w 2}\left(r^{6}-r\right), \quad r=\min \left(\frac{\tilde{\nu}}{\tilde{S} \kappa^{2} d^{2}}, r_{\lim }\right)
$$


The constants $c_{b 1}, c_{b 2}, c_{v 1}, c_{w 1}, c_{w 2}, c_{w 3}, \kappa$ and $r_{\text {lim }}$ used in the above definitions are taken from [24]. It is worth noting that the Reynolds stresses are proportional to the trace-less mean strain rate tensor according to the Boussinesq eddy viscosity assumption.

\section{Harmonic Balance RANS Solver}

Wind turbine aerodynamics consist of unsteady but temporally periodic flows usually due to the pitching blades or yawed wind regimes. In these cases, the conservative variables can be written in terms of a truncated Fourier series up to a predefined number of harmonics by:

$$
\mathbf{U}^{*}\left(x, y, t_{i}\right)=\mathbf{A}_{0}(x, y)+\sum_{n=1}^{N}\left[\mathbf{A}_{n}(x, y) \cos \left(\omega n t_{i}\right)+\mathbf{B}_{n}(x, y) \sin \left(\omega n t_{i}\right)\right]
$$

where $i=1: 2 N+1, \omega$ is the fundamental frequency of excitation and $\mathbf{A}_{0}, \mathbf{A}_{n}$ and $\mathbf{B}_{n}$ are the Fourier series coefficients defined in terms of the conservative variables. The Fourier series is truncated in a way that the flow variables are computed and stored at $2 N+1$ equally-spaced sub-time levels over a single period. Based on Equation (8) and using a discrete Fourier transform, the Fourier series coefficients can be determined from the solutions stored at each sub-time level as shown below:

$$
\mathbf{U}^{*}=\mathbf{E} \hat{\mathbf{U}}
$$

In a similar fashion, the conservative variables at the sub-time levels can be determined using the inverse discrete Fourier transform:

$$
\hat{\mathbf{U}}=\mathbf{E}^{-1} \mathbf{U}^{*}
$$

The discrete Fourier transform, $\mathbf{E}$, and its inverse, $\mathbf{E}^{-1}$, are both square matrices. Looking back at the unsteady governing equations in the time-domain, we have:

$$
\frac{d}{d t}(\mathcal{V} \mathbf{U})+\mathbf{Q}_{C}(\mathbf{U})+\mathbf{Q}_{V}(\mathbf{U})-\mathbf{Q}_{D}(\mathbf{U})=0
$$

where $\mathcal{V}$ denotes the control volume. The convective terms, $\mathbf{Q}_{C}(\mathbf{U})$, are evaluated at the cell centers by averaging the conservative variables at the corresponding cell vertex. A compact stencil central difference formulation [25] is used for the viscous terms, $\mathbf{Q}_{V}(\mathbf{U})$, which slightly reduces the odd-even decoupling in the solution field. However, the flux balance at each vertex still requires a robust artificial dissipation term, $\mathbf{Q}_{D}(\mathbf{U})$. The original JamesonSchmidt-Turkel (JST) formulation [21] is utilized to obtain the "scalar" artificial dissipation, which blends second and fourth differences of the conservation variables where the latter provides the background dissipation that is essential for stability and convergence toward steady state. However, the use of a 
low-speed preconditioning mechanism, which will be discussed in the following section, makes it inevitable to have a "matrix-valued" numerical viscosity that is scaled by the preconditioning matrix. In the original Jameson scheme, the dissipation or smoothing terms for the second and fourth differences in the artificial dissipation model are defined using a pressure switch. Finally, the system of semi-discretized initial-value ODEs is marched temporally toward a steady state solution with an explicit five-stage Runge-Kutta scheme in which the viscous terms are frozen at the first stage and the artificial dissipation terms are evaluated only at the odd stages. The coefficients of the five-stage Runge-Kutta scheme and the blending coefficients for the artificial dissipation terms [26] are given below:

$$
\begin{gathered}
A_{1}=\frac{1}{4}, \quad A_{2}=\frac{1}{6}, \quad A_{3}=\frac{3}{8}, \quad A_{4}=\frac{1}{2}, \quad A_{5}=1 \\
B_{1}=1, \quad B_{2}=0, \quad B_{3}=\frac{14}{25}, \quad B_{4}=0, \quad B_{5}=\frac{11}{25}
\end{gathered}
$$

The theoretical limit of the CFL number is 2.8 for the present Jameson scheme with RK5 and the CFL number for all steady as well as unsteady cases are set to $95 \%$ of this limit. Also, the coefficients of the second and fourth difference artificial dissipation terms are taken to be $\frac{1}{4}$ and $\frac{1}{64}$, respectively [21].

Using the harmonic balance method, as explained herein, the governing equations of (1) are rewritten similar to equation (11) in the semi-discrete form for all sub-time levels. The time-derivative term in equation (11) is then replaced by a pseudo-spectral operator $\mathbf{D}$, that gives:

$$
\mathbf{D}\left(\mathcal{V} \mathbf{U}^{*}\right)+\mathbf{Q}_{C}\left(\mathbf{U}^{*}\right)+\mathbf{Q}_{V}\left(\mathbf{U}^{*}\right)-\mathbf{Q}_{D}\left(\mathbf{U}^{*}\right)=0
$$

where the rest of the left-hand-side terms are the convective, viscous and artificial dissipation terms evaluated at each sub-time level. The pseudo-spectral operator is defined as

$$
\frac{d}{d t} \approx \mathbf{D}=\frac{d \mathbf{E}^{-1}}{d t} \mathbf{E}
$$

In order to obtain the steady state solution at each sub-time level, a "pseudotime" derivative term is added to the harmonic balance equation of (14) which enables the use of the solution algorithm explained earlier in this paper. This equation now reads:

$$
\frac{\partial}{\partial \tau}\left(\mathcal{V} \mathbf{U}^{*}\right)+\mathbf{D}\left(\mathcal{V} \mathbf{U}^{*}\right)+\mathbf{Q}_{C}\left(\mathbf{U}^{*}\right)+\mathbf{Q}_{V}\left(\mathbf{U}^{*}\right)-\mathbf{Q}_{D}\left(\mathbf{U}^{*}\right)=0
$$

where $\tau$ is the pseudo time, which enables us to march the above equation to steady state where the pseudo-time term vanishes, similar to our time-domain governing equations of (11).

As shown earlier by Hall et al. [13], an explicit treatment of the pseudospectral term in an explicit time-marching scheme can cause instabilities for 
the numerical solver. While non-reflecting far-field boundaries typically remedy these stability issues for low reduced frequencies, extra care must be given to cases with higher reduced frequencies or when too many harmonics are retained. In the present study, the CFL number constraint that was initially proposed by van der Weide et al. [27] is used to stabilize the explicit solver for a wide range of reduced frequencies and number of harmonics. The adapted stabilization technique introduces excitation frequency and the number of harmonics $(N)$ into the calculation of the pseudo-time-step where

$$
\Delta \tau=\frac{\mathrm{CFL} \mathcal{V}}{\|\lambda\|+\omega N \mathcal{V}}
$$

and $\|\lambda\|$ is taken to be the maximum of the spectral radii of the flux Jacobian matrices in all directions. The implementation of the present harmonic balance solver in wind turbine applications was presented by Howison and Ekici $[12,28,16]$.

\section{Low-Speed Preconditioning}

At low Mach numbers and on a fixed grid, convective waves that are traveling at speed $u$ become much slower than the acoustic waves that are traveling at $u+c$. What happens mathematically is that this disparity between the eigenvalues of the Jacobian matrices stiffens the governing equations, destabilizes the solution, slows down the convergence and degrades the accuracy by resulting in unbalanced amounts of artificial dissipation. Also, when an explicit timemarching method is used to obtain a steady state solution, the local time step depends on the eigenvalues of the flux Jacobian matrices, and the aforementioned disparity in wave speeds substantially impairs the convergence rate. To overcome these problems, low-speed preconditioning methods have been introduced which basically scale the acoustic wave speeds to be more on the order of the convective wave speeds.

As stated earlier, Venkateswaran and Merkle [8] originally presented the turbulent preconditioning using the $k$ - $\epsilon$ turbulence model for reacting and nonreacting turbulent flow problems. However, due to the inter-dependence of turbulence model equations, the resulting preconditioning system did not lead to real eigenvalues, which is not desirable in the framework of explicit timemarching schemes. This is not the case for the one-equation Spalart-Allmaras turbulence model which is used in the present RANS solver. Therefore, the extension of the original preconditioning methodology is straightforward.

\subsection{Preconditioning Matrix}

In this paper, a fully coupled viscous preconditioning of RANS equations is developed to tackle the convergence, stability and accuracy problems of solving an essentially incompressible flow with a compressible flow solver. The low-speed preconditioning mechanism fully incorporates the Spalart-Allmaras turbulence 
model in order to increase the efficiency of the preconditioner in resolving convergence deceleration due to the essential incompressibility of the flow regime. First, consider the two-dimensional RANS equations in primitive variables on a fixed grid using the primitive or non-conservation variables (pressure-velocityentropy) which are defined as $\mathbf{U}_{0}=[p, u, v, S, \tilde{\nu}]^{T}$. Thus, the quasi-linear form of this equation can be written as:

$$
\frac{\partial \mathbf{U}_{0}}{\partial t}+\mathbf{A}_{0} \frac{\partial \mathbf{U}_{0}}{\partial x}+\mathbf{B}_{0} \frac{\partial \mathbf{U}_{0}}{\partial y}=\mathbf{S}+\mathcal{L}_{\nu}\left(\mathbf{U}_{0}\right)
$$

where the Jacobian matrices of the flux vectors are given by:

$$
\mathbf{A}_{0}=\left[\begin{array}{ccccc}
u & \rho c^{2} & 0 & 0 & 0 \\
1 / \rho & u & 0 & 0 & 0 \\
0 & 0 & u & 0 & 0 \\
0 & 0 & 0 & u & 0 \\
0 & 0 & 0 & 0 & u
\end{array}\right] ; \quad \mathbf{B}_{0}=\left[\begin{array}{ccccc}
v & 0 & \rho c^{2} & 0 & 0 \\
0 & v & 0 & 0 & 0 \\
1 / \rho & 0 & v & 0 & 0 \\
0 & 0 & 0 & v & 0 \\
0 & 0 & 0 & 0 & v
\end{array}\right]
$$

The right hand side of equation (18) includes the vector of source terms, $\mathbf{S}$, and the differential operator for the viscous terms, $\mathcal{L}_{\nu}$. Based on the concept of artificial compressibility of Chorin [29] (for steady flows) the time derivative term can be multiplied by a preconditioning matrix, $\mathcal{P}_{0}^{-1}$, based on the primitive variables used. The quasi-linear set of the governing equations can then be multiplied by $\mathcal{P}_{0}$ to give

$$
\frac{\partial \mathbf{U}_{0}}{\partial t}+\mathcal{P}_{0} \mathbf{A}_{0} \frac{\partial \mathbf{U}_{0}}{\partial x}+\mathcal{P}_{0} \mathbf{B}_{0} \frac{\partial \mathbf{U}_{0}}{\partial y}=\mathcal{P}_{0}[\text { R.H.S. }]
$$

The preconditioning matrix, $\mathcal{P}_{0}$, can be easily derived in a primitive or symmetrizing set of variables based on the notion that the time-derivative of the pressure equation must be modified. The WSCM preconditioner of Colin et al. [10] not only takes care of the stagnation regions, which result in high local pressure disturbances, but also handles low Reynolds number regions which can lead to instabilities. It must be noted that the WSCM preconditioner uses primitive variables that are only limited to pressure, velocity and entropy. In this paper, we use a more generalized set of the primitive variables which includes the working variable of the Spalart-Allmaras turbulence model. This way, the general form of the symmetric preconditioner matrix can be written based on the modified set of variables, i.e. $\mathbf{U}_{0}=[p, u, v, S, \tilde{\nu}]^{T}$, as below:

$$
\mathcal{P}_{0}=\left[\begin{array}{ccccc}
\frac{\beta^{2}}{c^{2}} & 0 & 0 & 0 & 0 \\
0 & 1 & 0 & 0 & 0 \\
0 & 0 & 1 & 0 & 0 \\
0 & 0 & 0 & 1 & 0 \\
0 & 0 & 0 & 0 & 1
\end{array}\right]
$$

where $c$ is the local speed of sound and $\beta^{2}$ is the local free parameter that controls the amount of preconditioning. The preconditioned Jacobian matrices of the inviscid fluxes in primitive variables are then 


$$
\mathcal{P}_{0} \mathbf{A}_{0}=\left[\begin{array}{ccccc}
\frac{\beta^{2} u}{c^{2}} & \rho \beta^{2} & 0 & 0 & 0 \\
\frac{1}{\rho} & u & 0 & 0 & 0 \\
0 & 0 & u & 0 & 0 \\
0 & 0 & 0 & u & 0 \\
0 & 0 & 0 & 0 & u
\end{array}\right] ; \quad \mathcal{P}_{0} \mathbf{B}_{0}=\left[\begin{array}{ccccc}
\frac{\beta^{2} v}{c^{2}} & 0 & \rho \beta^{2} & 0 & 0 \\
0 & v & 0 & 0 & 0 \\
\frac{1}{\rho} & 0 & v & 0 & 0 \\
0 & 0 & 0 & v & 0 \\
0 & 0 & 0 & 0 & v
\end{array}\right]
$$

The eigenvalues for the system of preconditioned equations are now altered with the preconditioning parameter $\beta^{2}$ coming into play, changing the characteristics of the system. The eigenvalues for $\mathcal{P}_{0} \mathbf{A}_{0}$ and $\mathcal{P}_{0} \mathbf{B}_{0}$ on a fixed grid are given by

$$
\begin{aligned}
& \lambda_{A, 0}=u \quad ; \quad \lambda_{B, 0}=v \\
& \lambda_{A, \pm}=\frac{1}{2}\left[z u \pm \sqrt{z^{2} u^{2}+4 \beta^{2}\left(1-\frac{u^{2}}{c^{2}}\right)}\right] \\
& \lambda_{B, \pm}=\frac{1}{2}\left[z v \pm \sqrt{z^{2} v^{2}+4 \beta^{2}\left(1-\frac{v^{2}}{c^{2}}\right)}\right]
\end{aligned}
$$

where the subscripts $A$ and $B$ refer to the $\mathcal{P}_{0} \mathbf{A}_{0}$ and $\mathcal{P}_{0} \mathbf{B}_{0}$ matrices, respectively, and eigenvalues $\lambda_{A, 0}$ and $\lambda_{B, 0}$ each have the multiplicity of three while $\lambda_{A, \pm}$ and $\lambda_{B, \pm}$ correspond to the forward and backward acoustic waves in the respective directions. It is worth mentioning that in the case of a moving grid, the velocities in Equation (23) are changed to relative velocities that also include the grid velocities, i.e. $u-u_{g}$ and $v-v_{g}$, where $u_{g}$ and $v_{g}$ are the grid velocities in $x$ - and $y$-directions. The parameter $z$ in Equation (23) depends on the preconditioning parameter and is defined as

$$
z=1+\frac{\beta^{2}}{c^{2}}
$$

According to Turkel [30], while the entropy variables, $\mathbf{U}_{0}$, are useful for the basic formulation and analysis of the preconditioning mechanism, a more convenient set of primitive variables for viscous flows would be one that incorporates the temperature instead of entropy. This set would then be defined as $\mathbf{U}_{T}=[p, u, v, T, \tilde{\nu}]^{T}$. An important advantage is that the eigenvalues are invariant under variable transformations so that the eigenvalues of the system remain the same for any set of primitive variables. This is also true for the conservation form of the governing equations which is used in the present solver. The transformation matrices that take us from conservation to different sets of primitive variables and vice versa are given in the Appendix. Having the transformation matrices, we can derive the preconditioning matrix based on different sets of variables inside the solver by writing

$$
\mathcal{P}_{T}=\frac{\partial \mathbf{U}_{T}}{\partial \mathbf{U}_{0}} \mathcal{P}_{0} \frac{\partial \mathbf{U}_{0}}{\partial \mathbf{U}_{T}} \quad \text { or } \quad \mathcal{P}_{c}=\frac{\partial \mathbf{U}}{\partial \mathbf{U}_{0}} \mathcal{P}_{0} \frac{\partial \mathbf{U}_{0}}{\partial \mathbf{U}}
$$


The present RANS solver uses the governing equations in their conservation form which is similar to Equation (18) but are based on the set of conservation variables. Therefore, we have the quasi-linear form of the preconditioned set of RANS equations given by

$$
\frac{\partial \mathbf{U}}{\partial t}+\mathcal{P}_{c} \mathbf{A}_{c} \frac{\partial \mathbf{U}}{\partial x}+\mathcal{P}_{c} \mathbf{B}_{c} \frac{\partial \mathbf{U}}{\partial y}=\mathcal{P}_{c}[\text { R.H.S. }]
$$

where $\mathbf{A}_{c}$ and $\mathbf{B}_{c}$ are the Jacobian matrices of the flux vectors $\mathbf{F}$ and $\mathbf{G}$, respectively. Thus, in the framework of an explicit time-marching solver and using a blend of the second and fourth differences for the artificial dissipation, we have our residuals in conservation variables as

$$
\begin{aligned}
& \Delta \mathbf{U}=\Delta t\left\{\mathcal{P}_{c} \frac{\partial \mathbf{F}}{\partial x}+\mathcal{P}_{c} \frac{\partial \mathbf{G}}{\partial y}+\left(\left|\sigma\left(\mathcal{P}_{c} \mathbf{A}_{c}\right)\right| \frac{\partial \mathbf{U}}{\partial x}\right)_{x}+\left(\left|\sigma\left(\mathcal{P}_{c} \mathbf{A}_{c}\right)\right| \frac{\partial \mathbf{U}}{\partial x}\right)_{x x x}\right. \\
&\left.+\left(\left|\sigma\left(\mathcal{P}_{c} \mathbf{B}_{c}\right)\right| \frac{\partial \mathbf{U}}{\partial y}\right)_{y}+\left(\left|\sigma\left(\mathcal{P}_{c} \mathbf{B}_{c}\right)\right| \frac{\partial \mathbf{U}}{\partial y}\right)_{y y y}-\mathcal{P}_{c}[\text { R.H.S. }]\right\}
\end{aligned}
$$

where $\sigma\left(\mathcal{P}_{c} \mathbf{A}_{c}\right)$ and $\sigma\left(\mathcal{P}_{c} \mathbf{B}_{c}\right)$ are the spectral radii in the respective directions. By definition, the spectral radius is the largest eigenvalue when having a scalar artificial dissipation or the absolute of the Jacobian matrix when using a matrix dissipation model. Also $\left(\frac{\partial \mathbf{U}}{\partial x}\right)_{x}$ and $\left(\frac{\partial \mathbf{U}}{\partial x}\right)_{x x x}$ are the second and fourth differences that are applied to the set of conservation variables. Factoring out the preconditioning matrix that is given in conservation variables, we have

$$
\begin{aligned}
& \Delta \mathbf{U}=\Delta t \mathcal{P}_{c}\left\{\frac{\partial \mathbf{F}}{\partial x}+\frac{\partial \mathbf{G}}{\partial y}+\left(\mathcal{P}_{c}^{-1}\left|\sigma\left(\mathcal{P}_{c} \mathbf{A}_{c}\right)\right| \frac{\partial \mathbf{U}}{\partial x}\right)_{x}+\left(\mathcal{P}_{c}^{-1}\left|\sigma\left(\mathcal{P}_{c} \mathbf{A}_{c}\right)\right| \frac{\partial \mathbf{U}}{\partial x}\right)_{x x x}\right. \\
&\left.+\left(\mathcal{P}_{c}^{-1}\left|\sigma\left(\mathcal{P}_{c} \mathbf{B}_{c}\right)\right| \frac{\partial \mathbf{U}}{\partial y}\right)_{y}+\left(\mathcal{P}_{c}^{-1}\left|\sigma\left(\mathcal{P}_{c} \mathbf{B}_{c}\right)\right| \frac{\partial \mathbf{U}}{\partial y}\right)_{y y y}-[\text { R.H.S. }]\right\}
\end{aligned}
$$

Therefore, in the framework of a scalar artificial dissipation, $\sigma\left(\mathcal{P}_{c} \mathbf{A}_{c}\right)$ and $\sigma\left(\mathcal{P}_{c} \mathbf{B}_{c}\right)$ will be replaced by

$$
\begin{aligned}
\left|\sigma\left(\mathcal{P}_{c} \mathbf{A}_{c}\right)\right| & =\left|\frac{1}{2}\left[z u+\sqrt{z^{2} u^{2}+4 \beta^{2}\left(1-\frac{u^{2}}{c^{2}}\right)}\right]\right| \\
\left|\sigma\left(\mathcal{P}_{c} \mathbf{B}_{c}\right)\right| & =\left|\frac{1}{2}\left[z v+\sqrt{z^{2} v^{2}+4 \beta^{2}\left(1-\frac{v^{2}}{c^{2}}\right)}\right]\right|
\end{aligned}
$$

The presence of the $\mathcal{P}_{c}^{-1}$ matrix in the artificial dissipation terms gives a matrix-valued artificial dissipation even though we are using a scalar dissipation model, thereby altering the amount of artificial dissipation each equation has based on the preconditioning parameter. The vector of residuals in conservation 
variables has the changes in density as its first term while in the incompressible flow regime the density essentially remains constant. Therefore, the common practice is to calculate the residuals in terms of the primitive variables. For this reason and as discussed earlier, we have chosen the set of variables that includes the temperature, i.e. $\mathbf{U}_{T}=[p, u, v, T, \tilde{\nu}]^{T}$. Thus, using the respective transformation matrix, we have

$$
\begin{array}{r}
\Delta \mathbf{U}_{T}=\Delta t\left(\frac{\partial \mathbf{U}_{T}}{\partial \mathbf{U}} \mathcal{P}_{c}\right)\left\{\frac{\partial \mathbf{F}}{\partial x}+\frac{\partial \mathbf{G}}{\partial y}+\left(\mathcal{P}_{c}^{-1}\left|\sigma\left(\mathcal{P}_{c} \mathbf{A}_{c}\right)\right| \frac{\partial \mathbf{U}}{\partial x}\right)_{x}+\cdots\right. \\
\left.+\left(\mathcal{P}_{c}^{-1}\left|\sigma\left(\mathcal{P}_{c} \mathbf{B}_{c}\right)\right| \frac{\partial \mathbf{U}}{\partial y}\right)_{y}+\cdots\right\}
\end{array}
$$

where

$$
\Delta \mathbf{U}=\left[\begin{array}{c}
\Delta(\rho) \\
\Delta(\rho u) \\
\Delta(\rho v) \\
\Delta(\rho E) \\
\Delta(\rho \tilde{\nu})
\end{array}\right] \quad \rightarrow \quad \Delta \mathbf{U}_{T}=\left[\begin{array}{c}
\Delta(p) \\
\Delta(u) \\
\Delta(v) \\
\Delta(T) \\
\Delta(\tilde{\nu})
\end{array}\right]
$$

Therefore, in an existing solver based on the conservation variables, we first modify the eigenvalues of the system based on Equation (23) and pre-multiply the final artificial dissipation term by the inverse of the preconditioning matrix in the conservation form. Secondly, the total residual in the conservation variables is calculated based on the original procedure which is then transformed to the residuals in primitive variables using Equation (32) below. These residuals are used to update the primitive variables which are then used to calculate the updated conservation variables. It is worth mentioning that the transformed preconditioning matrix that is given in Equation (30) can be written as

$$
\frac{\partial \mathbf{U}_{T}}{\partial \mathbf{U}} \mathcal{P}_{c}=\frac{\partial \mathbf{U}_{T}}{\partial \mathbf{U}_{0}} \frac{\partial \mathbf{U}_{0}}{\partial \mathbf{U}}\left[\frac{\partial \mathbf{U}}{\partial \mathbf{U}_{0}} \mathcal{P}_{0} \frac{\partial \mathbf{U}_{0}}{\partial \mathbf{U}}\right]=\frac{\partial \mathbf{U}_{T}}{\partial \mathbf{U}_{0}} \mathcal{P}_{0} \frac{\partial \mathbf{U}_{0}}{\partial \mathbf{U}}
$$

Ultimately, all of the matrix multiplications are performed symbolically, and the general form of the preconditioning matrices are implemented into the code to reduce the computational cost and increase the accuracy of the numerical solver.

\subsection{Preconditioning Control Parameter}

The main challenge in the implementation and use of low-speed preconditioning is the choice of the free parameter $\beta$ that is on the order of the local velocity, making the $(1,1)$ element of the preconditioning matrix on the order of $\mathcal{O}\left(M^{2}\right)$. This parameter is defined based on the modifications of Turkel [31] and Colin et al. [10] as

$$
\beta^{2}=\min \left[c^{2}, \max \left(M_{0}^{2}, M^{2}, \epsilon \frac{|\Delta p|}{\rho c^{2}}, \alpha_{v i s c}\right) * c^{2}\right]
$$


where $M_{0}$ is a limiting Mach number to avoid a singular preconditioner matrix in the vicinity of solid walls and $M$ is the local Mach number. The pressure switch, which controls the amount of preconditioning near stagnation points, is defined by Darmofal and Siu [32] as the maximum of pressure variations in all constituent cells controlled by a case-dependent free parameter, $\epsilon$. Finally, for turbulent computations where viscosity dominated flow regimes cause the system of equations to become elliptic, Colin et al. [10] have studied the effects of some different controlling parameters, $\alpha_{v i s c}$, that are listed in Table 1 and are used in this work.

Table 1: Different controlling parameters for the viscous preconditioner [10].

\begin{tabular}{cccc}
\hline & Venkateswaran and Merkle [8] & Turkel [31] & Colin et al. [10] \\
\hline$\alpha_{\text {visc }}$ & $\left(\gamma \frac{\nu_{l} / P r_{l}+\nu_{t} / P r_{t}}{\Delta h}\right)^{2}$ & $K_{2} M_{\infty}^{2}$ & $M_{\text {isen }}^{2}$ \\
\hline
\end{tabular}

The $K_{2}$ parameter of the Turkel Preconditioner can take values between 0.5 to 1.0 while for viscous cases, it is suggested [31] to be tuned at higher values, e.g. as high as 5.0. Also, the Venkateswaran and Merkle [8] viscous parameter includes the viscosity and Prandtl numbers in laminar and turbulent states that are subscripted by corresponding letters. As shown by Colin et al. [10], the use of a local isentropic Mach number to control the amount of preconditioning inside the boundary layer or any other viscosity-dominated regions can lead to acceptable stability and convergence improvements. The latter approach is used in this study to account for viscous effects in our fully coupled low-speed preconditioner.

As presented earlier, in the framework of the harmonic balance method, the time derivative is approximated over sub-time level solutions using a pseudospectral operator, and an additional pseudo-time derivative is added to drive the solution to steady state. These approximations are utilized to drive out the transient errors and can be preconditioned to achieve an optimal convergence rate. Originally, Venkateswaran and Merkle [8] proposed an unsteady preconditioning mechanism for implicit and dual-time stepping methods in which an unsteady Mach number is added to the low-speed preconditioner (LSP). Potsdam et al. [33] have presented an improvement for unsteady scaling of the preconditioning matrix while more recently, Sachdev et al. [34] have extended the work of Potsdam et al. [33] to utilize the mixed unsteady and steady preconditioning parameter. The basic idea is that an unsteady Mach number scaling is added to the controlling parameter of the LSP such that

$$
\beta_{\text {uns }}^{2}=\min \left[c^{2}, \max \left(M_{0}^{2}, M^{2}, \epsilon \frac{|\Delta p|}{\rho c^{2}}, \alpha_{v i s c}, M_{u n s}^{2}\right) * c^{2}\right]
$$

where $M_{\text {uns }}$ is defined as

$$
M_{u n s}=\frac{\mathcal{L}_{u}}{\pi c \Delta t}
$$


where $\mathcal{L}_{u}$ is an unsteady length scale that controls the amount of mixed preconditioning. The characteristic length, $\mathcal{L}_{u}$, is typically taken as the physical dimension of the geometrical configuration that is being modeled which is basically a scale which represents the lowest wave number [35]. It can be seen that for small time-steps or very high excitation frequencies, the unsteady scale completely turns off the preconditioner, and thus, there is a locally-determined mixed-strategy between steady and no preconditioning conditions.

Additionally, and in the framework of the harmonic balance method, while the choice of the preconditioning parameter based on equation (33) leads to a robust preconditioning process for steady problems, Campobasso et al. [36] have shown that this form of preconditioning leads to an excessive amount of artificial dissipation that can negatively impact the convergence rate. Therefore, in the present study, the mixed-preconditioning strategy [36] for the harmonic balance solution is used which essentially brings an unsteady factor into the calculation of the preconditioning parameter. In fact, for the unsteady viscous low-speed preconditioning that is developed in this paper, the original viscous steady preconditioner that incorporates the turbulence model is used to calculate the preconditioned eigenvalues that would give the local time steps, and the unsteady $\beta_{\text {uns }}^{2}$ parameter is used to calculate the artificial dissipation terms. The unsteady viscous preconditioner with the incorporated Spalart-Allmaras turbulence equation is also used in preconditioning the total residuals in the framework of the explicit time-marching scheme.

\section{Boundary Conditions}

In the present study, the boundary conditions are applied at the far-field of the computational domain as well as on surface of the airfoil. At the far-field, characteristic theory is utilized to calculate the free stream and extrapolated values, where the speed of sound and the normal component of the velocity are defined via the Riemann invariants. Thus, the entropy and tangential component of the velocity are set to the free-stream values for the case of an incoming flow while the entropy and the tangential component of the velocity are set using extrapolated values for outgoing flows. Additionally, an adiabatic wall boundary as well as a no-slip condition (for viscous flows) are imposed at the airfoil surface. Since the characteristics of the system of equations are affected by the low-speed preconditioning, the boundary condition treatments are also changed for these cases. In this paper, the simplified boundary conditions of Turkel et al. [3] are used where velocity, pressure and density values at the boundary are defined as:

$$
\mathbf{u}_{b}=\mathbf{u}_{i / o}, \quad p_{b}=p_{o / i}, \quad \rho_{b}=\rho_{i / o}+\frac{p_{b}-p_{i / o}}{c^{2}}
$$

In the above equation, the subscripts $i$ and $o$ refer to incoming or outgoing flows which can be used interchangeably depending on the boundary acting as an inflow or outflow, respectively. In the following section, the unsteady viscous low-speed preconditioning is applied to steady and periodically unsteady 
cases in which obtaining an accurate steady solution in a reasonable number of iterations would be a challenge using the original compressible fluid flow solver.

\section{Steady Low-Speed Preconditioning Results}

To evaluate the effectiveness of the low-speed preconditioning in dealing with low-subsonic flows, first a series of steady simulations are performed. In an earlier work [37], the inviscid flow over the NACA0012 airfoil at zero angle of attack and very low Mach numbers has been studied. Although the case was inviscid, it served as a very good example demonstrating how the accuracy of the compressible flow solver is negatively affected by the disparity of the eigenvalues in an incompressible flow regime and how this can be remedied using a robust low-speed preconditioning algorithm. In this paper, we first look at the classical benchmark problem of turbulent flow over a flat-plate with zero pressure gradient. For this case, the effect of the present viscous low-speed preconditioning is studied considering the convergence rate and boundary layer predictions. The results are compared to those obtained from the CFL3D solver [38] and the WSCM preconditioner [10] where the turbulence model has been excluded in the preconditioning process. As another steady test case, the flow over an S809 airfoil is studied. Although prediction of the stall and post-stall behaviors of the S809 airfoil using a RANS solver without a transition model is difficult, using a robust viscous low-speed preconditioning proves to be effective in significantly accelerating the convergence to the steady state while shifting the surface pressure results toward the experimental data. For all these computations, the limiting Mach number is taken to be $M_{0}=10^{-6}$ which can switch off the controlling parameter for low Mach numbers to avoid a singular preconditioning matrix. Also, the free parameter for the pressure gradient switch, which controls the amount of preconditioning near stagnation points, is varied from very small to large values. Note that the convergence rate improves as this parameter is decreased, and numerical results have indicated an insensitivity to values less than $\epsilon<1 e-4$, and is thus taken to be $\epsilon=1.0^{-4}$.

\subsection{Turbulent Flow Over a Flat Plate with Zero Pressure Gradient}

The turbulent flow over a flat plate with zero pressure gradient is a classical benchmark problem that is used here to validate the numerical solution and evaluate the robustness of the present viscous preconditioner. The numerical results are compared with those from the CFL3D code developed at NASA in the 1980s. For this purpose, the turbulent flow is simulated along a 2-meter long flat plate at a Mach number of 0.2 . Since the Mach number is in the incompressible region, using a compressible flow solver without the low-speed preconditioning tool can cause instability, inaccuracy and slow convergence as explained earlier. The Reynolds number based on the length of the plate is $R e=5$ million at half plate length, i.e. $x=1$. For the Spalart-Allmaras turbulence model, the value of the working variable, $\tilde{\nu}$, at the far-field is set to be three times that of the reference viscosity, i.e. $\tilde{\nu}=3 \nu_{\infty}$. 
One challenging problem here is the generation of a decent grid with appropriate element quality close to the leading and trailing edges of the flat plate. This was addressed [12] as one of the deteriorating factors when using an O-grid that was constructed using an elliptic grid generator. To avoid this, a modified Joukowski transformation is used to generate an O-grid based on the flat plate geometries. The grid in the cross-flow plane $\xi-\eta$ is generated using the Joukowski transformation, and grid stretching is added to cluster nodes close to the leading and trailing edges of the flat plate. This method, which was originally proposed by Marconi [39], leads to "semi-conformal" grids with decent and adjustable resolution near the surface of the flat plate. Although not shown here, an O-grid with $321 \times 129$ nodes is found to give mesh independent results for the present case. The grid extends well over 50 plate length in all directions, and the minimum spacing at the wall is $y=3.2 \times 10^{-6}$, which corresponds to a $y^{+}$value of approximately 0.6 along the length of the flat plate. The viscous grid in the trailing edge region is shown in Figure 1. It is worth mentioning that the results from the CFL3D code are obtained using a rectangular $545 \times 385$ grid where the flat plate is placed at the bottom side.

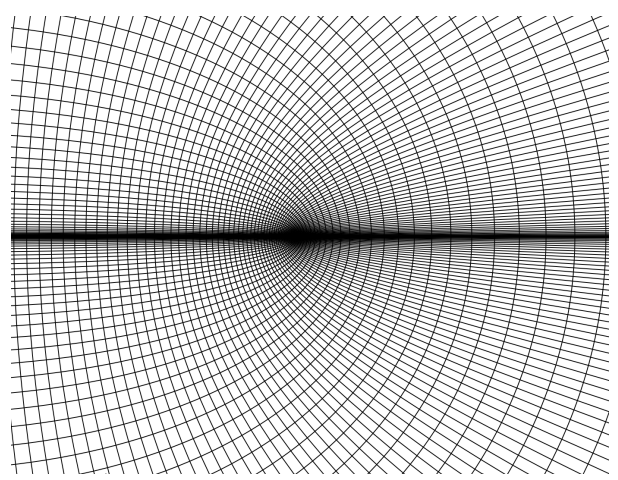

Figure 1: Semi-conformal viscous O-grid at the downstream end of the flat plate.

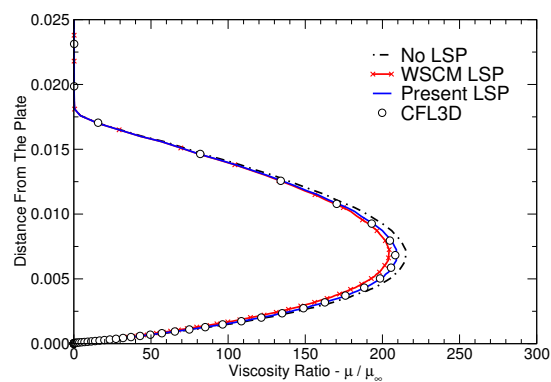

(a)

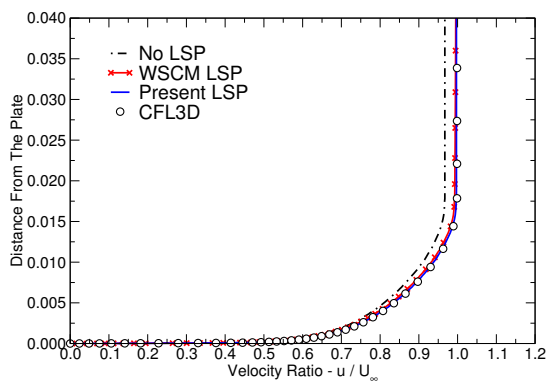

(b)

Figure 2: Flow over a flat plate with $R e=10$ million, $M=0.2$ : (a) Eddy viscosity to free-stream viscosity ratio, $\mu_{t} / \mu_{\infty}$, at $x=0.97$, (b) velocity ratio profile, $u / U_{\infty}$, at $x=0.97$. 


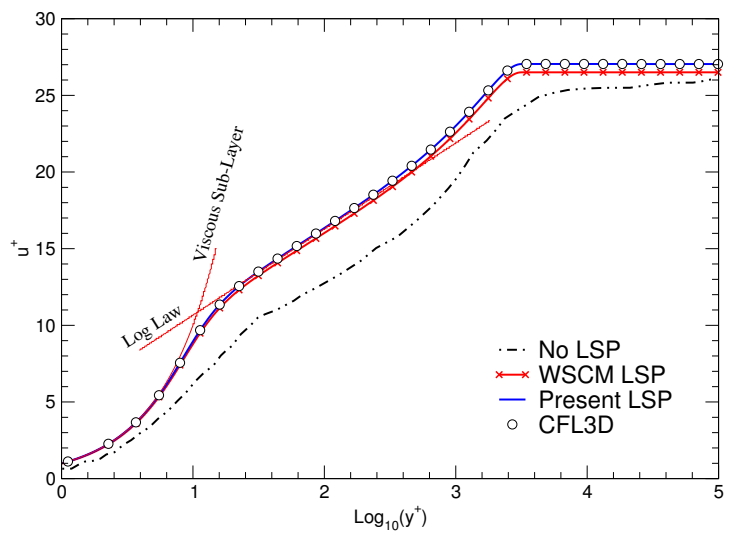

Figure 3: Law of the wall at $x=0.97$ including the theoretical profiles in the viscous sub-layer and log-law region for flow over a flat plate with $R e=10$ million, $M=0.2$.

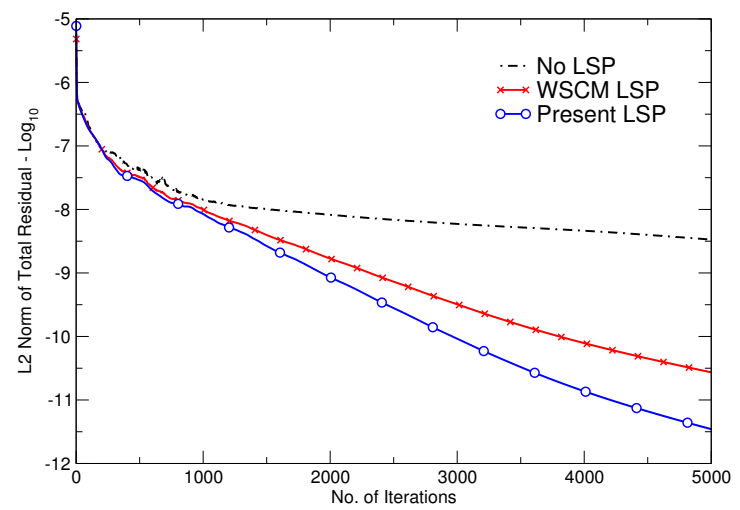

Figure 4: Convergence rates with WSCM and present viscous preconditioning vs. no preconditioning for the flow over a flat plate with $R e=10$ million, $M=0.2$.

In an earlier work, Howison and Ekici [12] saw some differences between their skin friction values along the upper surface of the plate in comparison with those of CFL3D. With the use of the preconditioning, the present method gives skin friction values that perfectly match CFL3D results as well as the theory. We suspect that this was mainly due to the inclusion of the turbulence equation or its working variable in the low-speed preconditioning algorithm. The effects of the viscous low-speed preconditioning (LSP) can be seen in the numerical results that are compared to those from the WSCM preconditioner without incorporating the turbulence model. Also, note that the grid quality that was used in [12] was not as good as the one used herein. The ratio of the eddy to the free-stream viscosity and the non-dimensional velocity profile at $x=0.97$ using WSCM and present preconditioners are compared with the results of the CFL3D solver and are shown in Figure 2. A good agreement is obtained with other solvers for 
the viscosity ratio. Except for a small difference in the maximum value, the velocity profile is identical to that obtained from the CFL3D solver. As can be clearly seen, the results from the WSCM preconditioner are slightly off while the ones with the present viscous preconditioner perfectly match. Additionally, the law of the wall at $x=0.97$ is shown in Figure 3, which perfectly matches the results of CFL3D and the theoretical profiles in the viscous sub-layer and log-law regions.

Finally, the convergence rate of the solver with and without low-speed preconditioning is presented in Figure 4 for the total residual of the RANS equations. While the numerical accuracy is preserved, the slow convergence is improved with the aid of present low-speed preconditioning. The effect of the present low-speed preconditioner, which fully couples the turbulence model to the rest of the governing equations is more pronounced compared to the WSCM preconditioner or the case without any low-speed preconditioning. It is worth mentioning that the CPU time per iteration is increased by about $9 \%-10 \%$ for the preconditioned case while the required number of iterations to reach the same level of accuracy is reduced for about $65 \%$ for the WSCM and $70 \%$ for the present LSP. The residual of the turbulent conservation variable, $\rho \tilde{\nu}$, is also plotted in Figure 5 for the cases with WSCM and present LSP as well as without the LSP. As can be seen in Figure 5, the best convergence rate is achieved using the present LSP while there is a plateau in the convergence of the non-preconditioned case.

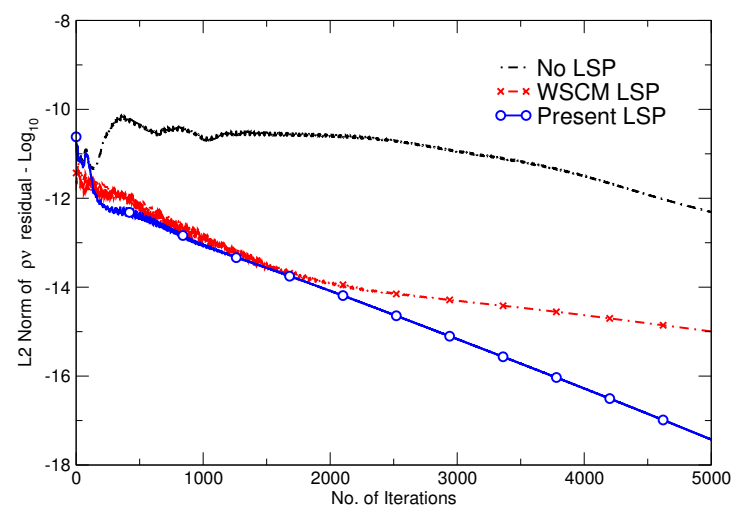

Figure 5: Convergence rates for the turbulent conservation variable, $\rho \tilde{\nu}$ with WSCM and present LSP vs. no preconditioning for the flow over a flat plate at $M=0.2$.

\subsection{Steady Flow Past S809 Airfoil}

The last steady case considered here is the turbulent flow over the S809 airfoil. This case is studied for Mach number of $M=0.1$ and Reynolds number of one million. Here, two cases are analyzed for angles of attack set to AOA = $4.1^{\circ}$ and $\mathrm{AOA}=12.2^{\circ}$. The data of Ramsay et al. [40] from wind tunnel tests at the Ohio State University are used for comparison. An O-grid that 
contains $401 \times 139$ nodes in the circumferential and normal directions is used and has a minimum spacing of $4 \times 10^{-6}$ near the wall, which corresponds to $y^{+} \approx 0.2$. A close-up view of the O-grid is shown in Figure 6 . The grid extends approximately 50 chord lengths in all directions, and although not shown here, grid independence studies have shown only a $2 \%$ variation in the lift and moment coefficients when the grid resolution is doubled.

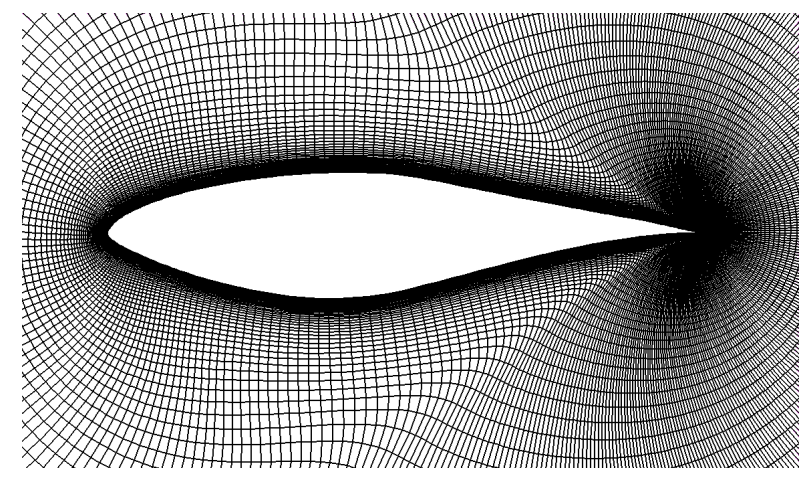

Figure 6: O-grid for S809 airfoil with $401 \times 139$ nodes.

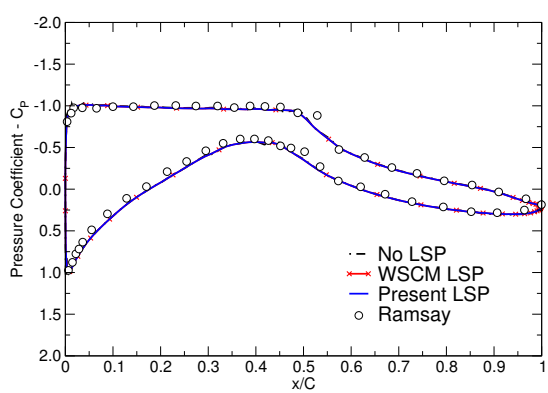

(a)

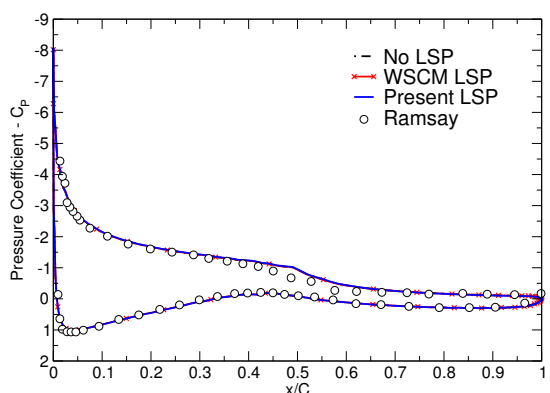

(b)

Figure 7: Surface pressure coefficients for steady S809 airfoil with and without low-speed preconditioning. (a) $\mathrm{AOA}=4 \cdot 1^{\circ}$; (b) $\mathrm{AOA}=12.2^{\circ}$.

In order to validate the obtained numerical results, a comparison of the computed surface pressure coefficients with the experimental data is presented in Figure 7. As can be seen, there is good agreement with the experimental results of Ramsay et al. [40] although certain flow features can only be captured with a transition model [16]. As shown in Figure 8, the convergence is significantly improved with the present low-speed preconditioner compared to the WSCM LSP and the case without any preconditioning. As discussed earlier, although there is only a slight difference between the preconditioned and non-preconditioned results in terms of the surface coefficient, the convergence is significantly accelerated using the present viscous low-speed preconditioning that effectively 
incorporates the turbulence model in the preconditioning mechanism.

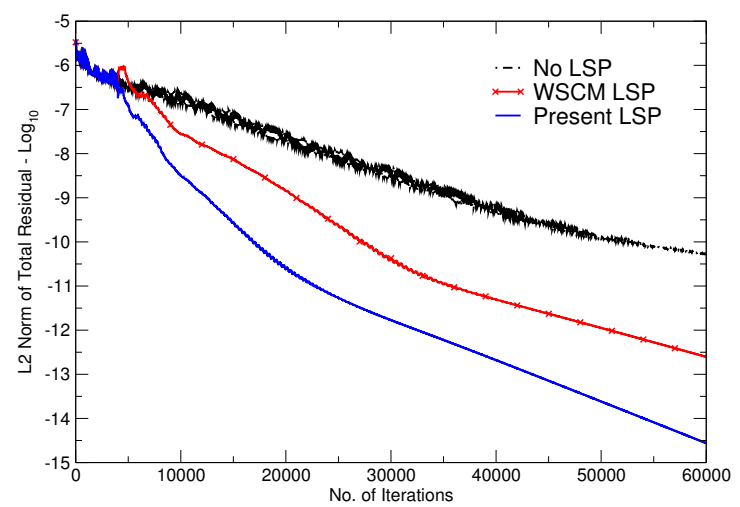

Figure 8: Steady convergence rates for S809 airfoil with and without low-speed preconditioning for the case with $\mathrm{AOA}=4.1^{\circ}, \mathrm{M}=0.1$ and $\mathrm{Re}=1$ million.

\section{Unsteady Viscous Low-Speed Preconditioning Results}

The implementation of a robust viscous low-speed preconditioning was discussed earlier, and the effects of this preconditioner on the accuracy and the convergence for steady flow were studied through two turbulent flow cases. Now that the present viscous low-speed preconditioning technique is found to be effective in steady cases, we turn our attention to the unsteady low-subsonic flows over pitching airfoils using the harmonic balance RANS solver. We first look at two pitching cases for unsteady NACA 0012 airfoil in subsonic and lowsubsonic regimes. Next, turbulent flow over a pitching NACA 0015 airfoil in the low-subsonic regime is studied. As will be shown later, the convergence of the solver is significantly accelerated with the use of the present viscous low-speed preconditioning.

\subsection{Unsteady NACA 0012 Airfoil Results}

The first unsteady case considered is the turbulent flow over a pitching NACA 0012 airfoil at two different nominal angles of attack and pitch amplitudes. The two test cases are evaluated by the experimental data of McAlister et al. [41], and the settings are given in Table 2.

Table 2: Pitching NACA 0012 airfoil test settings.

\begin{tabular}{|c|c|c|c|c|c|}
\hline $\begin{array}{c}\text { Case } \\
\#\end{array}$ & $\begin{array}{c}\text { Mach } \\
\text { Number, } \\
\text { M }\end{array}$ & $\begin{array}{c}\text { Mean Angle } \\
\text { of Attack, } \\
\alpha_{m}\end{array}$ & $\begin{array}{c}\text { Pitching } \\
\text { Amplitude, } \\
\alpha_{p}\end{array}$ & $\begin{array}{c}\text { Reduced } \\
\text { Frequency, } \\
k\end{array}$ & $\begin{array}{c}\text { Reynolds } \\
\text { Number } \\
\text { Re }\end{array}$ \\
\hline 1 & 0.301 & $4.93^{\circ}$ & $4.99^{\circ}$ & 0.198 & $3.91 \times 10^{6}$ \\
\hline 2 & 0.301 & $7.978^{\circ}$ & $4.91^{\circ}$ & 0.199 & $3.89 \times 10^{6}$ \\
\hline
\end{tabular}


These cases are previously studied numerically by Yang et al. [42] using an Euler method, and as shown by McCroskey and Pucci [43], the dynamic stall onset is at $\alpha_{\max }=14^{\circ}$. Therefore, no dynamic stall occurs in either case. Both of these unstalled pure pitching oscillation cases are at $M=0.3$, which is the limit of compressibility. Since we usually switch off the low-speed preconditioner around $M=0.3$, there would be no significant improvement in the accuracy or convergence when enabling the LSP mechanism. However, in order to study the effects of present turbulent preconditioner that is also enhanced with the mixed-preconditioning mechanism, we also run the cases with $M=0.1$ with the rest of the flow settings being the same as those of [41].

In all of these cases, the airfoil pitches about the quarter-chord from the leading edge. An O-grid (shown in Figure 9) with $281 \times 129$ nodes in circumferential and normal directions was generated that gave a $y^{+}$of about 0.6. The grid extends approximately 120 chord lengths in all directions, and although not presented here, grid independence studies have shown less than $4 \%$ variation in the unsteady lift and moment coefficients when the grid resolution is doubled.

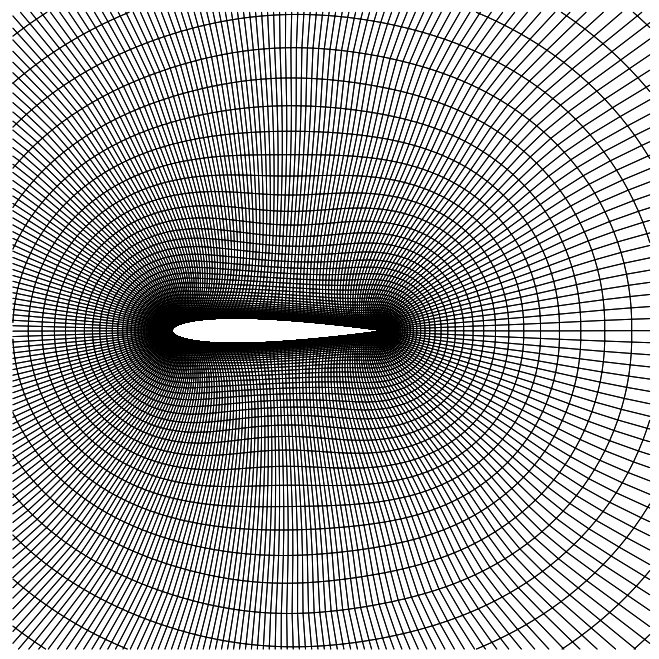

Figure 9: NACA 0012 O-grid with $281 \times 129$ nodes.

Mode convergence for HB solutions is achieved when the solutions do not change with additional harmonics. It is worth mentioning that mode convergence is achieved for all cases with and without preconditioning with 2 to 3 harmonics. Therefore, as shown in Figure 10 for the surface pressure coefficients of the mean flow using 2 and 3 harmonics, two harmonics for the HB solver is found to be sufficient for both cases that are considered herein.

Figure 11 depicts the pressure coefficient $C_{p}$ over the upper and lower surfaces of the airfoil at mean angle of attack compared to the experimental data [41], which are only available for the upper surface. It is worth mentioning that in Case $1, \omega t=0$ corresponds to $\alpha=4.93^{\circ}$ in pitch-up, and in Case 


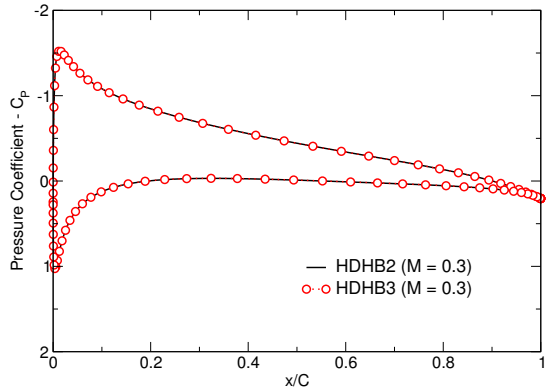

(a)

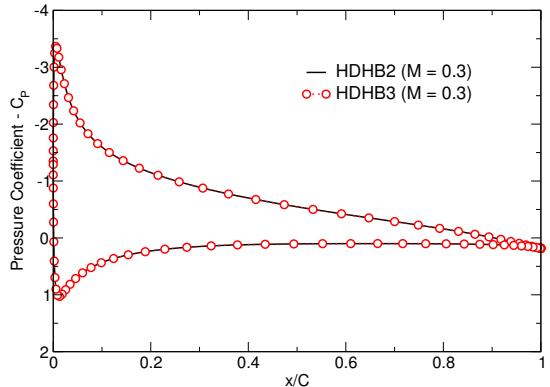

(b)

Figure 10: Mode convergence study using the surface pressure coefficients of the mean flow (a) Case 1; (b) Case 2.

2 , it corresponds to $\alpha=7.978^{\circ}$ in pitch-up. As can be seen in Figure 11, there is a fair agreement with the experimental data. In order to evaluate the performance of the present RANS solver at low-subsonic flow regimes, we have reduced the Mach number down to $M=0.1$ for both cases. Since the flow lies in the essentially incompressible regime, the present turbulent unsteady low-speed preconditioner is utilized. As discussed earlier, the isentropic Mach number is used as the viscous controlling parameter so that local and global preconditioning is applied inside and outside of the boundary layer, respectively. Moreover, a mixed-preconditioning strategy with a choice of a well-tuned unsteady length is used to handle the unsteadiness that is present in the harmonic solution. As discussed earlier in Section 4, the characteristic length for the unsteady LSP is usually taken to be the physical dimensions of the geometrical configuration being modeled [8]. Therefore, the characteristic length is set to $\mathcal{L}_{u}=1.0$ for this unsteady case. Numerical results from the original cases and cases with $M=0.1$ with and without LSP are compared against each other and experimental data [41] (see Figure 11).

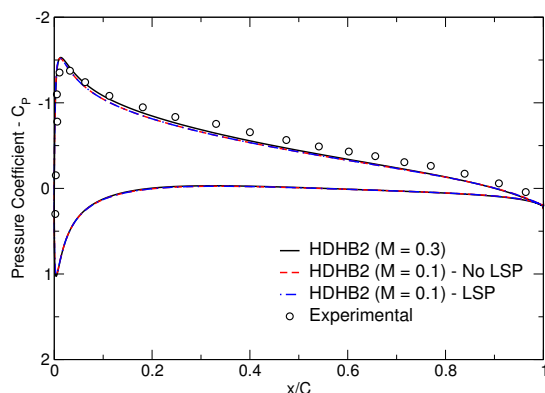

(a)

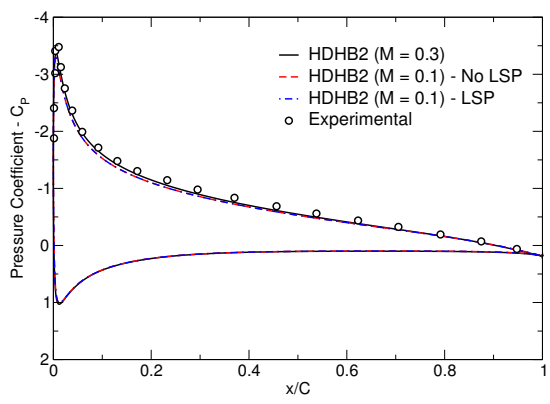

(b)

Figure 11: Surface pressure coefficients of the mean flow with original settings and with $M=0.1$ compared against experimental data [41], (a) Case 1; (b) Case 2.

Next, the harmonic unsteady surface pressure coefficients are presented for 
cases 1 and 2 with both original settings and with $M=0.1$. The numerical results for the zeroth harmonic of the unsteady pressure coefficient, $C_{p 0}$, are normalized using the mean angle of attack, while the results for the first harmonic of the unsteady surface pressure coefficient are normalized using the pitch amplitude. It is worth mentioning that the first harmonic of the unsteady pressures has real and imaginary or cosine and sine parts that are plotted separately. Numerical harmonic components of the surface pressure coefficients are compared against the experimental data of McCroskey and Pucci [43] and incompressible linear theory [43] for Cases 1 and 2 with $M=0.3, M=0.1$ without LSP and $M=0.1$ with present turbulent unsteady LSP. These plots are shown in Figures 12 through 14.

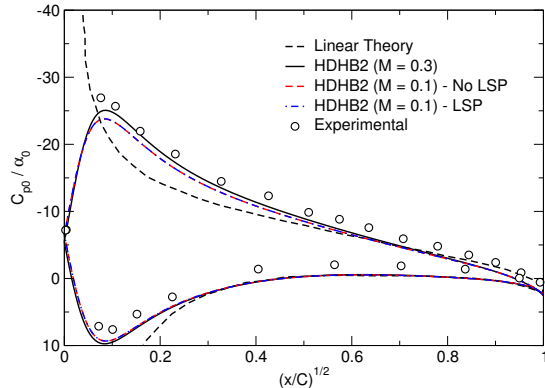

(a)

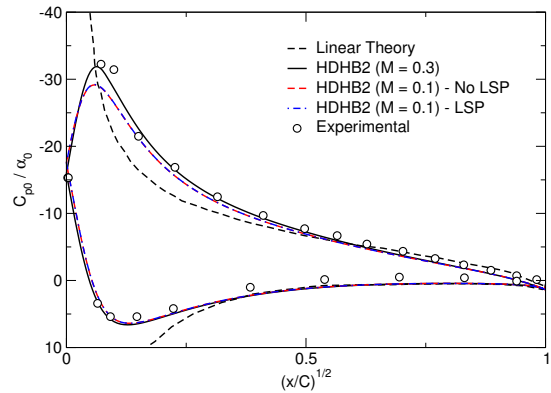

(b)

Figure 12: Zeroth harmonic of unsteady surface pressure coefficients compared against experimental data [43] and linear theory, (a) Case 1; (b) Case 2.

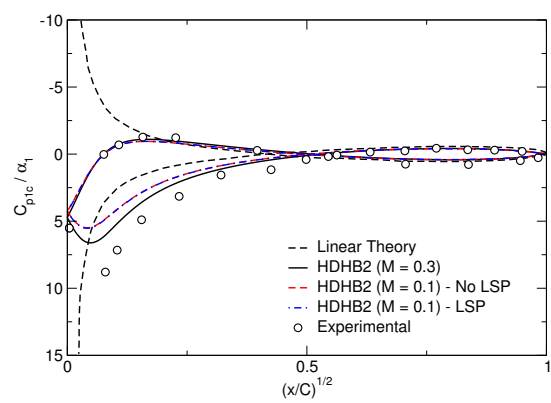

(a)

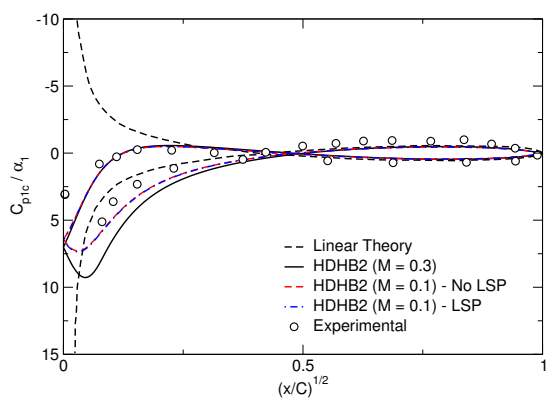

(b)

Figure 13: Real part of the first harmonic of unsteady surface pressure coefficients compared against experimental data [43] and linear theory, (a) Case 1; (b) Case 2.

Since the main difference between two cases that are studied here is the mean angle of attack, a similar pattern can be seen in the unsteady results. However, since the mean angle of attack for Case 2 is greater than that of Case 1, greater fluctuating pressure loads are exhibited in Case 2. This behavior is not seen in the linear theory. Also, there is a very good agreement with the numerical and experimental data for the original cases, and as expected, the magnitude of 


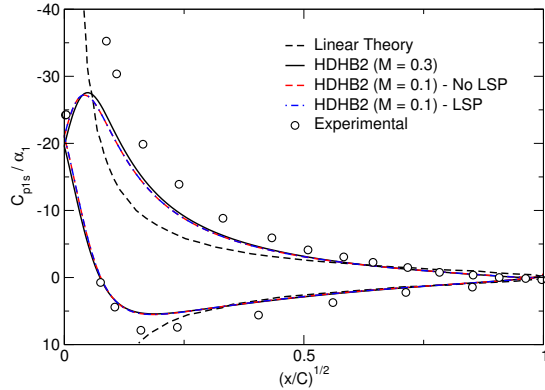

(a)

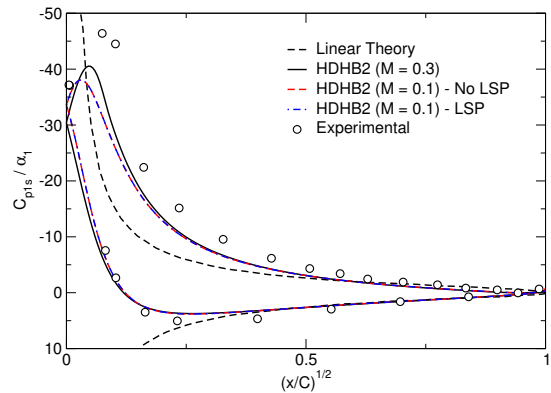

(b)

Figure 14: Imaginary part of the first harmonic of unsteady surface pressure coefficients compared against experimental data [43] and linear theory, (a) Case 1; (b) Case 2.

the unsteady pressures is slightly reduced for cases with $M=0.1$. In all these plots, the unsteady pressure results were presented versus $(x / C)^{1 / 2}$, and the square-root of the non-dimensional length is used to stretch out the solutions in the vicinity of the leading-edge region where major variations happen.

As shown earlier, present turbulent unsteady LSP was applied to the pitching NACA 0012 cases with reduced Mach number that lies in the essentially incompressible flow regime. However, no significant difference was shown in the numerical results for cases with and without LSP. To further the analysis, the convergence history for the numerical solver in the case of $M=0.1$ is studied in terms of the total residuals, and the results are shown in Figure 15. As can be seen, for both cases the present turbulent LSP that is enhanced with the mixedpreconditioning strategy is capable of accelerating the convergence significantly. Also, the unpreconditioned cases start to plateau for the total residuals in the order of $10^{-11}$ while the present LSP avoids any levelling-off and presents guaranteed convergence down to machine accuracy. It is worth mentioning that the CPU time per iteration is increased by about $8 \%$ for the preconditioned case using WSCM preconditioner and about 10\%-11\% using the present LSP with and without unsteady modifications. Additionally, the required number of iterations to reach the same level of accuracy is reduced for about $50 \%$ using the present LSP with mixed-mechanism.

The residual of the turbulent conservation variable, $\rho \tilde{\nu}$, is also plotted in Figure 16 for the cases with WSCM and present LSP (with and without unsteady limiter) as well as without the LSP. As can be seen, the best convergence rate is achieved using the present LSP with unsteady limiter. Moreover, the required number of iterations to reach the same level of accuracy for the turbulent conservation variable is reduced for about $40 \%$ for the WSCM LSP, $57 \%$ for the present LSP without unsteady modification and $60 \%$ for the present LSP with unsteady limiter.

As discussed before, an important advantage of the present viscous lowspeed preconditioner is to accelerate the convergence to steady solution, which improves even further with the addition of the mixed-preconditioning mecha- 


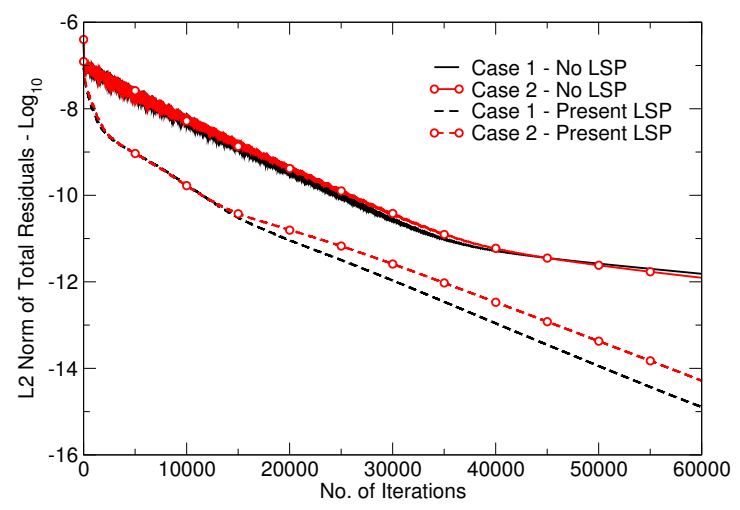

Figure 15: Convergence rates for pitching NACA 0012 airfoil tests, with and without turbulent unsteady LSP for cases given in Table 2 at $M=0.1$.

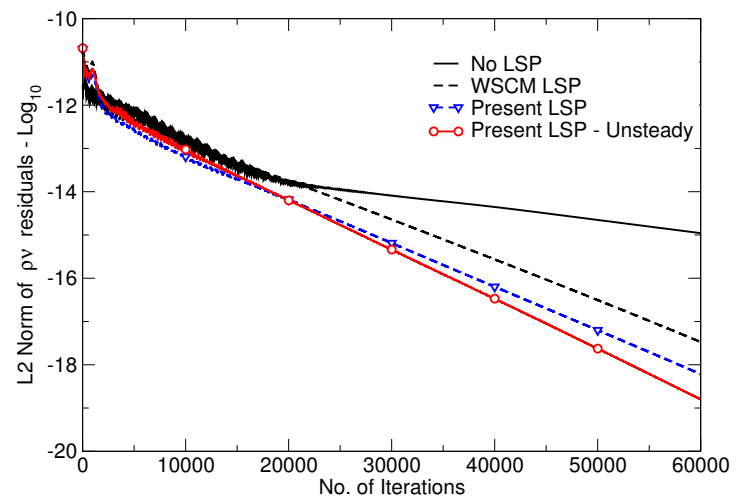

Figure 16: Convergence rates of the turbulent conservation variable, $\rho \tilde{\nu}$, for pitching NACA 0012 airfoil test (Case 1) using present LSP with and without unsteady modifications compared to WSCM LSP and no LSP cases.

nism for unsteady flows. It can be seen that the present LSP can significantly accelerate the convergence although its effect on the numerical solution was not very significant. This proves that the present LSP with the unsteady mixedpreconditioning mechanism can be a valuable tool for the convergence acceleration in the flow cases that are essentially in the incompressible regime.

\subsection{Turbulent Flow Over a Pitching NACA 0015 Airfoil}

The second unsteady case considered is the low-speed turbulent flow past a NACA 0015 airfoil that undergoes a harmonic pitching motion. The mean angle of attack is taken to be $10^{\circ}$ with a pitching amplitude of $4.0^{\circ}$. The airfoil pitches about the quarter-chord from the leading edge, and the free-stream Mach number and the Reynolds number are 0.2 and 1.5 million, respectively. This case was also studied by Piskopakis [44] using both time-accurate and harmonic balance solvers with the Wilcox's $k$ - $\omega$ turbulence model [45]. An O-grid (shown in Figure 17) with $287 \times 161$ nodes in circumferential and normal directions was 
generated that gave a $y^{+}$of 0.4 . The grid extends approximately 85 chord lengths in all directions, and although not shown here, grid independence studies have shown less than $3 \%$ variation in the unsteady lift and moment coefficients when the grid resolution is doubled. The reduced frequency, $k=b \omega / U_{\infty}$, based on the half-chord length is taken to be 0.2 for this case. The number of harmonics that are retained in the HB solver is varied between 1 and 3 , and mode convergence is achieved for three harmonics.

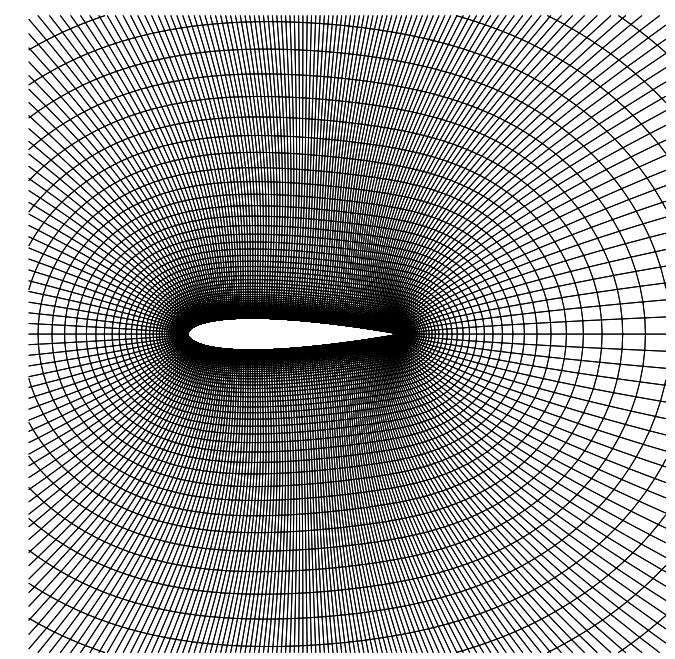

Figure 17: NACA 0015 O-grid with $287 \times 161$ nodes.

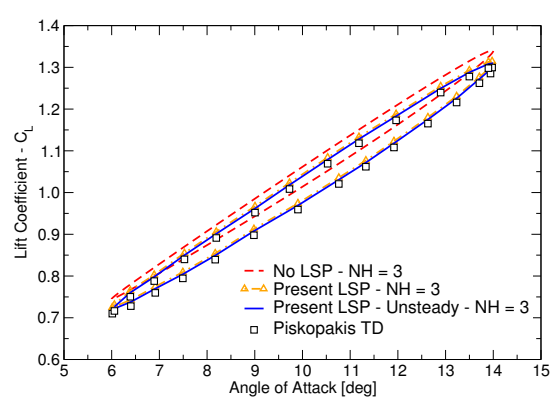

(a)

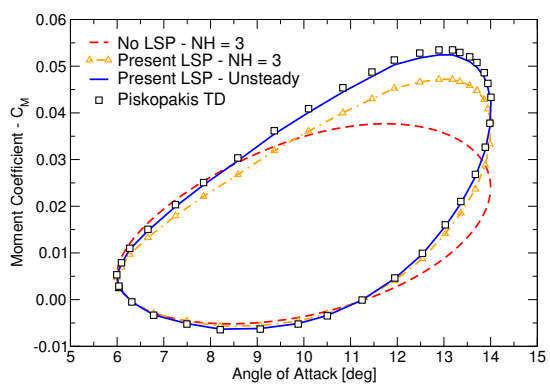

(b)

Figure 18: Hysteresis cycle of the moment and lift coefficients for the pitching NACA 0015 airfoil using the present viscous LSP with and without mixed-preconditioning (results taken from snapshots after 60,000 iterations), (a) Lift Coefficient; (b) Moment Coefficient.

The present viscous low-speed preconditioner is enhanced with the mixedpreconditioning mechanism that incorporates the unsteadiness of the low-subsonic flow regime. To show the effect, the numerical results are presented for the current viscous low-speed preconditioner with and without mixed-LSP. The nu- 
merical results in terms of the lift and moment coefficients are given in Figure 18. Note that in all cases, 3 harmonics are retained in the HB solver, and the results are compared with those obtained using the time-accurate solver of Piskopakis [44]. The solutions are taken from the snapshots after 60,000 iterations where the results from the present LSP with the unsteady limiter are converged down to machine accuracy (see Figure 20). As can be clearly seen, the present LSP can effectively improve the accuracy of the numerical solution by accelerating the convergence rate. As discussed earlier in Section 4, the characteristic length for the unsteady LSP is usually taken to be the physical dimensions of the geometrical configuration being modeled [8], while it can also be challenging sometimes to find a well-tuned characteristic length to achieve maximum convergence acceleration. For the present unsteady case, although not shown here, it has been seen that better convergence is achieved for the characteristic lengths set to a larger value than the physical dimension of the airfoil and in fact, the best acceleration is achieved using the mixed-preconditioning mechanism with $\mathcal{L}_{u}=1.5$ set as the unsteady length scale. It is also worth noting that the numerical results are identical for all these cases using the present LSP with different unsteady limiters and the characteristic length only affects the amount of convergence acceleration.

In order to gain further insight into the effectiveness of the present viscous preconditioner along with the mixed-preconditioning mechanism, the real part of the first harmonic of the surface pressure coefficient is presented in Figure 19. It can be seen that the best agreement with time-accurate solver is achieved when the mixed-preconditioning mechanism is used for the present viscous lowspeed preconditioner. Once again the numerical results for all cases are taken from the snapshots after 60,000 iterations.

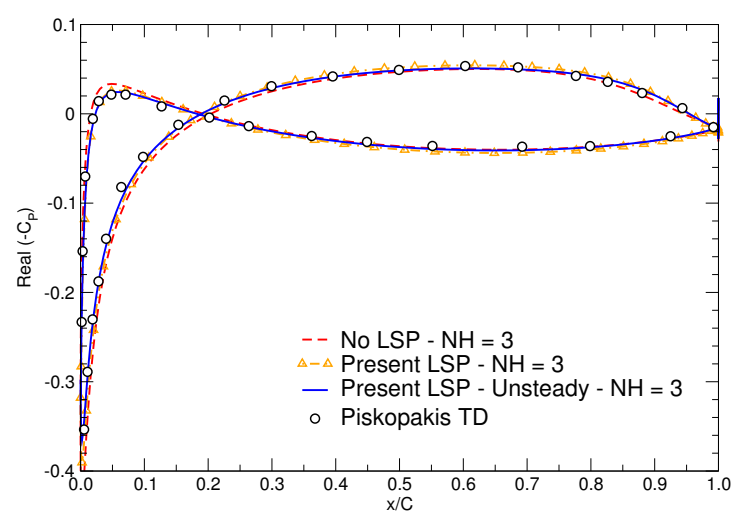

Figure 19: First harmonic of the unsteady surface pressure coefficients (real part) for the flow past NACA0015 (results taken from snapshots after 60, 000 iterations).

Next, the convergence rates are compared to the non-preconditioned case. As shown in Figure 20, the present low-speed preconditioner can effectively accelerate the convergence. Also, a better convergence acceleration is achieved using a mixed-preconditioning mechanism that incorporates the effects of the 


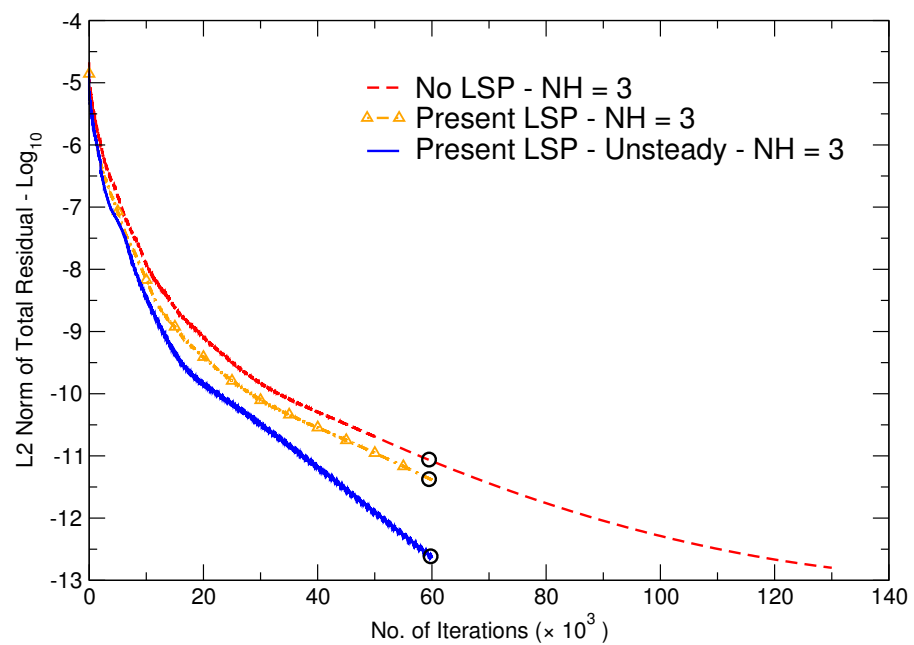

Figure 20: Steady convergence rate for the flow past NACA 0015 using the present viscous LSP with and without mixed-preconditioning mechanism. Location of the snapshots plotted in Figure 18 are shown with circles.

flow unsteadiness. In an earlier work [37], the authors have compared the convergence rates for the cases with 1, 2 and 3 harmonics retained in the harmonic balance solutions. As discussed in [37], the effect of the present unsteady viscous LSP is more pronounced in cases with a higher number of harmonics. This may be due to the effect of the harmonic stabilization used for the CFL criterion given in Equation (17).

To demonstrate the importance of converging the solution close to machine accuracy, we next plot the lift and moment coefficients hysteresis in Figure 21. As one can see, the solutions for no LSP and present LSP (unsteady) become identical when no LSP case is run for 130,000 iterations. Also shown on the same figure are the solutions for no LSP after 60,000 iterations where the total residual was driven down to $1 \times 10^{-11}$. This clearly demonstrates the need to fully converge the solution for accurate results. Although the fully converged numerical results from the non-preconditioned case and using the present LSP (unsteady) are identical (see Figure 21 below), the present LSP with the unsteady limiter has led to about $55 \%$ decrease in the required number of iterations in order to achieve the same level of accuracy.

\section{Conclusions}

A viscous unsteady low-speed preconditioner is developed that fully incorporates the Spalart-Allmaras turbulence model into the preconditioning mechanism. To the best of our knowledge, this is the first time that the preconditioning algorithm is fully coupled with the Spalart-Allmaras turbulence model through a complete set of primitive variables and in the framework of a harmonic balance RANS solver. The viscous LSP is also enhanced with a mixed-preconditioning 


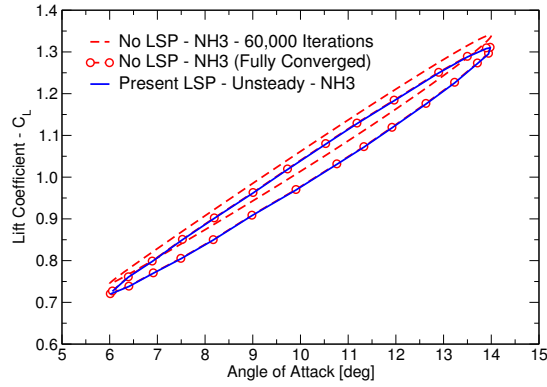

(a)

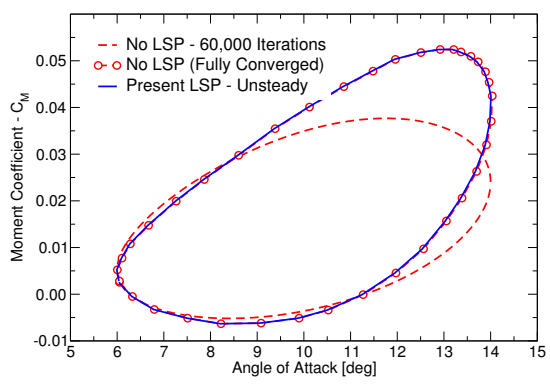

(b)

Figure 21: Hysteresis cycle of the moment and lift coefficients for the pitching NACA 0015 airfoil. Intermediate and fully converged No LSP results are taken from the solutions after 60, 000 and 130, 000 iterations, respectively and the present LSP (unsteady) results are taken after 60,000 iterations, (a) Lift Coefficient; (b) Moment Coefficient.

mechanism that further improves the ability of the preconditioner in accelerating the convergence of the harmonic solution. The present LSP is applied to steady and unsteady two-dimensional cases including the turbulent flow over a flat-plate with zero pressure gradient and flow around pitching NACA 0012 and 0015 airfoils. Although the CPU time per iteration is increased for about 9\%-11\% using the present LSP, there has been a $50 \%-60 \%$ decrease in the required number of iterations to achieve the same level of accuracy as with the cases without low-speed preconditioning.

\section{Acknowledgements}

This material is based upon work partially supported by the National Science Foundation under grant no: CBET-1150332. The authors greatly appreciate the support provided.

\section{Appendix}

Conservation and primitive variables sets and the corresponding transformation matrices:

$$
\mathbf{U}_{0}=\left[\begin{array}{c}
p \\
u \\
v \\
S \\
\tilde{\nu}
\end{array}\right] \quad ; \quad \mathbf{U}_{T}=\left[\begin{array}{c}
p \\
u \\
v \\
T \\
\tilde{\nu}
\end{array}\right] \quad ; \quad \mathbf{U}=\left[\begin{array}{c}
\rho \\
\rho u \\
\rho v \\
\rho E \\
\rho \tilde{\nu}
\end{array}\right]
$$




$$
\begin{aligned}
\frac{\partial \mathbf{U}_{T}}{\partial \mathbf{U}_{0}}= & {\left[\begin{array}{ccccc}
1 & 0 & 0 & 0 & 0 \\
0 & 1 & 0 & 0 & 0 \\
0 & 0 & 1 & 0 & 0 \\
\frac{(\gamma-1) T}{\gamma p} & 0 & 0 & \frac{T}{\gamma p} & 0 \\
0 & 0 & 0 & 0 & 1
\end{array}\right] ; \frac{\partial \mathbf{U}_{0}}{\partial \mathbf{U}_{T}}=\left[\begin{array}{ccccc}
1 & 0 & 0 & 0 & 0 \\
0 & 1 & 0 & 0 & 0 \\
0 & 0 & 1 & 0 & 0 \\
1-\gamma & 0 & 0 & \frac{\gamma p}{T} & 0 \\
0 & 0 & 0 & 0 & 1
\end{array}\right] } \\
\frac{\partial \mathbf{U}}{\partial \mathbf{U}_{0}}= & {\left[\begin{array}{ccccc}
\frac{1}{c^{2}} & 0 & 0 & -\frac{1}{c^{2}} & 0 \\
\frac{u}{c^{2}} & \rho & 0 & -\frac{u}{c^{2}} & 0 \\
\frac{v}{c^{2}} & 0 & \rho & -\frac{v}{c^{2}} & 0 \\
\frac{1}{\gamma-1}+\frac{\left(u^{2}+v^{2}\right)}{2 c^{2}} & \rho u & \rho v & -\frac{\left(u^{2}+v^{2}\right)}{2 c^{2}} & 0 \\
\frac{\tilde{\nu}}{c^{2}} & 0 & 0 & -\frac{\tilde{\nu}}{c^{2}} & \rho
\end{array}\right] } \\
\frac{\partial \mathbf{U}_{0}}{\partial \mathbf{U}}= & {\left[\begin{array}{cccccc}
\frac{(\gamma-1)\left(u^{2}+v^{2}\right)}{2} & u(1-\gamma) & v(1-\gamma) & (\gamma-1) & 0 \\
-\frac{u}{\rho} & \frac{1}{\rho} & 0 & 0 & 0 \\
-\frac{v}{\rho} & 0 & \frac{1}{\rho} & 0 & 0 \\
\frac{\left(u^{2}+v^{2}\right)(\gamma-1)}{2}-c^{2} & u(1-\gamma) & v(1-\gamma) & (\gamma-1) & 0 \\
-\frac{\tilde{\nu}}{\rho} & 0 & 0 & 0 & \frac{1}{\rho}
\end{array}\right] }
\end{aligned}
$$

\section{References}

[1] E. Turkel, Preconditioned methods for solving the incompressible and low speed compressible equations, Journal of Computational Physics 72 (2) (1987) 277-298.

[2] E. Turkel, A. Fiterman, B. Van Leer, Preconditioning and the limit to the incompressible flow equations, Tech. rep., DTIC Document (1993).

[3] E. Turkel, R. Radespiel, N. Kroll, Assessment of preconditioning methods for multidimensional aerodynamics, Computers \& Fluids 26 (6) (1997) 613634.

[4] Y.-H. Choi, C. L. Merkle, The application of preconditioning in viscous flows, Journal of Computational Physics 105 (2) (1993) 207-223.

[5] J. M. Weiss, W. A. Smith, Preconditioning applied to variable and constant density flows, AIAA Journal 33 (11) (1995) 2050-2057.

[6] B. Van Leer, W.-T. Lee, P. L. Roe, Characteristic time-stepping or local preconditioning of the Euler equations, in: 10th Computational Fluid Dynamics Conference, Vol. 1, 1991, pp. 260-282.

[7] F. Liu, X. Zheng, A strongly coupled time-marching method for solving the Navier-Stokes and k- $\omega$ turbulence model equations with multigrid, Journal of Computational Physics 128 (2) (1996) 289-300.

[8] S. Venkateswaran, L. Merkle, Analysis of preconditioning methods for the Euler and Navier-Stokes equations, Lecture series-von Karman Institute for Fluid Dynamics 3 (1999) B1-B155. 
[9] M. Campobasso, M. Yan, J. Drofelnik, A. Piskopakis, M. Caboni, Compressible Reynolds-Averaged Navier-Stokes analysis of wind turbine turbulent flows using a fully-coupled low-speed preconditioned multigrid solver, in: ASME Turbo Expo 2014: Turbine Technical Conference and Exposition, American Society of Mechanical Engineers, 2014, pp. V03BT46A010V03BT46A010.

[10] Y. Colin, H. Deniau, J.-F. Boussuge, A robust low speed preconditioning formulation for viscous flow computations, Computers \& Fluids 47 (1) (2011) $1-15$.

[11] M. S. Campobasso, M. H. Baba-Ahmadi, Analysis of unsteady flows past horizontal axis wind turbine airfoils based on harmonic balance compressible Navier-Stokes equations with low-speed preconditioning, Journal of Turbomachinery 134 (6) (2012) 061020.

[12] J. Howison, K. Ekici, Unsteady analysis of wind turbine flows using the harmonic balance method, AIAA Paper 2013-1107 (2013).

[13] K. C. Hall, J. P. Thomas, W. S. Clark, Computation of unsteady nonlinear flows in cascades using a harmonic balance technique, AIAA Journal 40 (5) (2002) 879-886.

[14] K. C. Hall, K. Ekici, J. P. Thomas, E. H. Dowell, Harmonic balance methods applied to computational fluid dynamics problems, International Journal of Computational Fluid Dynamics 27 (2) (2013) 52-67.

[15] H. Huang, K. Ekici, An efficient harmonic balance method for unsteady flows in cascades, Aerospace Science and Technology 29 (1) (2013) 144154 .

[16] J. Howison, K. Ekici, Dynamic stall analysis using harmonic balance and correlation-based $\gamma-\operatorname{Re}_{\theta t}$ transition models for wind turbine applications, Wind Energy 18 (12) (2015) 2047-2063.

[17] K. Ekici, R. E. Kielb, K. C. Hall, Aerodynamic asymmetry analysis of unsteady flows in turbomachinery, Journal of Turbomachinery 132 (1) (2010) 011006 .

[18] P. R. Spalart, S. R. Allmaras, A one equation turbulence model for aerodynamic flows, AIAA Paper 92-0439 (1992).

[19] J. Dacles-Mariani, G. G. Zilliac, J. S. Chow, P. Bradshaw, Numerical/experimental study of a wingtip vortex in the near field, AIAA Journal 33 (9) (1995) 1561-1568.

[20] J. Dacles-Mariani, D. Kwak, G. Zilliac, On numerical errors and turbulence modeling in tip vortex flow prediction, International Journal for Numerical Methods in Fluids 30 (1) (1999) 65-82. 
[21] A. Jameson, W. Schmidt, E. Turkel, Numerical solutions of the Euler equations by finite volume methods using Runge-Kutta time-stepping schemes, AIAA Paper 81-1259 (1981).

[22] R. C. Swanson, E. Turkel, On central-difference and upwind schemes, Journal of Computational Physics 101 (2) (1992) 292-306.

[23] E. Turkel, V. N. Vatsa, Effect of artificial viscosity on three-dimensional flow solutions, AIAA Journal 32 (1) (1994) 39-45.

[24] S. R. Allmaras, F. T. Johnson, P. R. Spalart, Modifications and clarifications for the implementation of the Spalart-Allmaras turbulence model, in: Seventh International Conference on Computational Fluid Dynamics (ICCFD7), 2012, pp. 1-11.

[25] L. Martinelli, A. Jameson, F. Grasso, A multigrid method for the NavierStokes equations, AIAA Paper 86-0208 (1986).

[26] K. Ekici, K. C. Hall, H. Huang, J. P. Thomas, Stabilization of explicit flow solvers using a Proper-Orthogonal-Decomposition technique, AIAA journal 51 (5) (2013) 1095-1104.

[27] E. Van Der Weide, A. Gopinath, A. Jameson, Turbomachinery applications with the time spectral method, AIAA Paper 2005-4905 (2005).

[28] J. Howison, K. Ekici, Wind turbine dynamic stall analysis using harmonic balance and correlation-based transition models, AIAA Paper 2013-2951 (2013).

[29] A. J. Chorin, A numerical method for solving incompressible viscous flow problems, Journal of Computational Physics 2 (1) (1967) 12-26.

[30] E. Turkel, Robust low speed preconditioning for viscous high lift flows, AIAA Paper 2002-0962 (2002).

[31] E. Turkel, V. N. Vatsa, Local preconditioners for steady and unsteady flow applications, ESAIM: Mathematical Modelling and Numerical Analysis 39 (03) (2005) 515-535.

[32] D. Darmofal, K. Siu, A robust multigrid algorithm for the Euler equations with local preconditioning and semi-coarsening, Journal of Computational Physics 151 (2) (1999) 728-756.

[33] M. A. Potsdam, V. Sankaran, S. A. Pandya, Unsteady low mach preconditioning with application to rotorcraft flows, AIAA Paper 2007-4473 (2007).

[34] J. Sachdev, A. Hosangadi, V. Sankaran, Improved flux formulations for unsteady low mach number flows, AIAA Paper 2012-3067 (2012).

[35] S. Venkateswaran, C. Merkle, Dual time stepping and preconditioning for unsteady computations, AIAA Paper 1995-0078 (1995). 
[36] M. Campobasso, A. Bonfiglioli, M. Baba-Ahmadi, Development of efficient and accurate CFD technologies for wind turbine unsteady aerodynamics, in: Proceedings of the Conference on Modeling Fluid Flow, J. Vad, ed., 14th Event of International Conference Series on Fluid Flow Technologies held in Budapest, Department of Fluid Mechanics, Budapest University of Technology and Economics, Vol. 2, 2009, pp. 879-886.

[37] R. Djeddi, K. Ekici, A turbulent low-speed preconditioner for unsteady flows about wind turbine airfoils, AIAA Paper 2015-3202 (2015).

[38] C. L. Rumsey, T. B. Gatski, Summary of EASM turbulence models in CFL3D with validation test cases, NASA/TM-2003-212431, June 2003.

[39] F. Marconi, The spiral singularity in the supersonic inviscid flow over a cone, AIAA Paper 83-1665 (1983).

[40] R. Ramsay, M. Hoffman, G. Gregorek, Effects of grid roughness and pitch oscillations on the s809 airfoil, Technical Paper 442-7817, NREL.

[41] K. McAlister, S. Pucci, W. McCroskey, L. Carr, An experimental study of dynamic stall on advanced airfoil sections. Volume 2. Pressure and force data, Defense Technical Information Center, 1982.

[42] S. Yang, S. Luo, F. Liu, H.-M. Tsai, Subsonic flow over unstalled pitching airfoil computed by Euler method, AIAA Paper 2006-3914 (2006).

[43] W. McCroskey, S. Pucci, Viscous-inviscid interaction on oscillating airfoils in subsonic flow, AIAA Journal 20 (2) (1982) 167-174.

[44] A. Piskopakis, Time-domain and harmonic balance turbulent Navier-Stokes analysis of oscillating foil aerodynamics, Ph.D. thesis, University of Glasgow (2014).

[45] D. C. Wilcox, Reassessment of the scale-determining equation for advanced turbulence models, AIAA Journal 26 (11) (1988) 1299-1310. 